\title{
A UNIFIED FRAMEWORK OF SAGE AND SONC POLYNOMIALS AND ITS DUALITY THEORY
}

\author{
LUKAS KATTHÄN, HELEN NAUMANN, AND THORSTEN THEOBALD
}

\begin{abstract}
We introduce and study a cone which consists of a class of generalized polynomial functions and which provides a common framework for recent non-negativity certificates of polynomials in sparse settings. Specifically, this $\mathcal{S}$-cone generalizes and unifies sums of arithmetic-geometric mean exponentials (SAGE) and sums of non-negative circuit polynomials (SONC). We provide a comprehensive characterization of the dual cone of the $\mathcal{S}$-cone, which even for its specializations provides novel and projection-free descriptions. As applications of this result, we give an exact characterization of the extreme rays of the $\mathcal{S}$-cone and thus also of its specializations, and we provide a subclass of functions for which non-negativity coincides with membership in the $\mathcal{S}$-cone.

Moreover, we derive from the duality theory an approximation result of non-negative univariate polynomials and show that a SONC analogue of Putinar's Positivstellensatz does not exist even in the univariate case.
\end{abstract}

\section{INTRODUCTION}

In recent years, several interrelated approaches for non-negative polynomials and for nonnegative exponential sums have been proposed, which are aimed at sparse settings. In [4], Chandrasekaran and Shah proposed (in the language of exponential sums/signomials) to consider sums of polynomial functions $f: \mathbb{R}_{+}^{n} \rightarrow \mathbb{R}$ of the form $\sum_{\alpha \in \mathcal{A}} c_{\alpha} \mathbf{x}^{\alpha}$ for a given set $\mathcal{A} \subseteq \mathbb{R}^{n}$ such that at most one term has a negative coefficient. Non-negativity of signomials can be characterized in terms of the arithmetic-geometric mean inequality, and deciding membership in the resulting cone (SAGE cone) can be formulated as a relative entropy program. In [13], Iliman and de Wolff proposed to consider sums of non-negative circuit polynomials on $\mathbb{R}^{n}$ (SONC polynomials). For a certain subclass of polynomials called ST-polynomials, deciding membership in the cone of SONC polynomials can be formulated in terms of the optimization subclass of geometric programs [7]. Murray, Chandrasekaran and Wierman [21] have shown that an adaption of the SAGE setting to $\mathbb{R}^{n}$ gives exactly the same cone of polynomials as the SONC cone. This yields a computationally tractable method to decide membership of arbitrary polynomials in the SONC cone using a relative entropy program.

While many aspects of these classes of polynomials are connected with open questions and research efforts, they clearly exhibit some fundamental structural phenomena adapted to sparse settings. For example, it was shown by Murray et al. [21] for the SAGE cone and by Wang [26] for the SONC cone that every polynomial in those cones has a cancellation-free representation. Generally, SAGE and SONC approaches can be combined with semidefinite approaches

Date: September 22, 2020. 
to polynomial optimization, see Karaca, Darivianakis et al. [15] or Averkov [3]. Moreover, by [3, Theorem 2.16] and its proof, the SONC cone is second-order-cone representable (but the size of the second-order formulation from that work depends on the actual values of the support vectors). For a practical algorithm to compute SONC bounds via second-order cone representations, see Magron and Wang [27].

The goal of the present paper is to provide a uniform framework which covers all these classes as well as some more general settings. Since non-negativity of a polynomial function $f\left(x_{1}, \ldots, x_{n}\right)$ on $\mathbb{R}_{+}^{n}$ is equivalent to non-negativity of $f\left(\left|x_{1}\right|, \ldots,\left|x_{n}\right|\right)$ on $\mathbb{R}^{n}$, we consider the more general functions $f: \mathbb{R}^{n} \rightarrow \mathbb{R} \cup\{\infty\}$ of the form

$$
f(\mathbf{x})=\sum_{\alpha \in \mathcal{A}} c_{\alpha}|\mathbf{x}|^{\alpha}+\sum_{\beta \in \mathcal{B}} d_{\beta} \mathbf{x}^{\beta}
$$

with sets of exponents $\mathcal{A} \subseteq \mathbb{R}^{n}, \mathcal{B} \subseteq \mathbb{N}^{n} \backslash(2 \mathbb{N})^{n}$, which also capture the signomial functions. Based on a subset of these functions, we define the $\mathcal{S}$-cone $C_{\mathcal{S}}(\mathcal{A}, \mathcal{B})$ which provides the common generalization of the cones mentioned above, see Definition 2.3. Its atomic functions are called $A G$ functions, which are functions of the form (1) with strong support conditions. The AG functions can be seen as a (non-polynomial) generalization of polynomials coming from the arithmetic-geometric inequality. Building upon the earlier work of the second and the third author [9] on the dual SONC cone, a particular focus is the structure and the use of the dual viewpoint.

Non-negative polynomials and polynomial optimization are ubiquitous in applications, and sparsity is one of the central structural properties that provides potential for efficient computation. Besides classical application in control theory and robotics (see, e.g., [2, 12] and the references therein), let us list the more recent applications of non-negative polynomials and polynomial optimization in the optimal power flow problem [14], collision avoidance [1] or shape-constrained regression [10].

Contributions. 1. We show that fundamental properties of the SAGE and/or the SONC cone also hold in the more general context of the $\mathcal{S}$-cone. In particular, every $f \in C_{\mathcal{S}}(\mathcal{A}, \mathcal{B})$ can be decomposed into a sum of non-negative AG functions whose supports are contained in the support of $f$. See Proposition 2.7, which unifies and generalizes the results of [21] for the SAGE cone and of $[26]$ for the SONC cone.

2. We provide a comprehensive characterization of the dual cone of the $\mathcal{S}$-cone, see Theorem 3.5. In particular, we provide projection-free characterizations in terms of AG functions supported on the particular class of reduced circuits. The characterizations of the dual cone go far beyond the characterizations of the dual SAGE cone from [4] and the dual SONC cone from [9], where the dual cones are described in terms of projections. Our proofs provide a uniform tool set for handling the various types of cones.

3. Based on the characterizations of the dual of the $\mathcal{S}$-cone, we provide several applications of the duality theory.

(a) We show that every sum $f$ of non-negative AG functions can be written as a sum of non-negative circuit functions whose supports are contained in the support of $f$. This unifies and generalizes the results from [21] for the SAGE cone and of [26] for the SONC cone. 
(b) We give an exact characterization of the extreme rays of the $\mathcal{S}$-cone. Even for the particular case of the SAGE cone, this characterization substantially sharpens the necessary conditions in [21].

(c) We show that not even in the univariate case, SONC polynomials do allow Putinar-type representations. This counterexample strengthens and simplifies the result of Dressler, Kurpisz and de Wolff [8], who have provided a multivariate counterexample.

(d) We give a characterization of a wide class of non-negative AG functions with simplex Newton polytopes. Using the dual $\mathcal{S}$-cone, this result unifies and generalizes the results from [13] and [21] and provides a simpler proof.

(e) As a final application of the dual $\mathcal{S}$-cone, we show that non-negative univariate polynomials can be approximated by SONC polynomials.

As further related work, let us mention the exploitation of sparsity and symmetries to derive specific SDP relaxations for polynomial optimization [16, 20, 24, 28, 29, 30].

\section{The $\mathcal{S}$-Cone}

In this section, we introduce $\mathrm{AG}$ functions and the $\mathcal{S}$-cone. We show that every non-negative function in the $\mathcal{S}$-cone has a cancellation-free representation (see Proposition 2.7) and characterize non-negativity of an AG function in terms of the relative entropy function (see Theorem 2.8).

Notation. Throughout the article we use the notations $\mathbb{N}=\{0,1,2,3, \ldots\}$ and $\mathbb{R}_{+}=\{x \in \mathbb{R}$ : $x \geq 0\}$. Moreover, for a finite subset $\mathcal{A} \subseteq \mathbb{R}^{n}$, denote by $\mathbb{R}^{\mathcal{A}}$ the set of $|\mathcal{A}|$-dimensional vectors whose components are indexed by the set $\mathcal{A}$.

Our main object of study are functions $f: \mathbb{R}^{n} \rightarrow \mathbb{R} \cup\{\infty\}$ of the form

$$
f(\mathbf{x})=\sum_{\alpha \in \mathcal{A}} c_{\alpha}|\mathbf{x}|^{\alpha}+\sum_{\beta \in \mathcal{B}} d_{\beta} \mathbf{x}^{\beta}
$$

where $\mathcal{A} \subseteq \mathbb{R}^{n}, \mathcal{B} \subseteq \mathbb{N}^{n} \backslash(2 \mathbb{N})^{n}$ are finite sets of exponents, $\left\{c_{\alpha}: \alpha \in \mathcal{A}\right\},\left\{d_{\beta}: \beta \in \mathcal{B}\right\} \subseteq \mathbb{R}$. Here we use the notations

$$
|\mathbf{x}|^{\alpha}=\prod_{j=1}^{n}\left|x_{j}\right|^{\alpha_{j}} \quad \text { and } \quad \mathbf{x}^{\beta}=\prod_{j=1}^{n} x_{j}^{\beta_{j}},
$$

and if one component of $\mathbf{x}$ is zero and the corresponding exponent is negative, then we set $|\mathbf{x}|^{\alpha}=\infty$.

For two finite sets $\emptyset \neq \mathcal{A} \subseteq \mathbb{R}^{n}, \mathcal{B} \subseteq \mathbb{N}^{n} \backslash(2 \mathbb{N})^{n}$, let

$$
\mathbb{R}[\mathcal{A}, \mathcal{B}]:=\operatorname{span}_{\mathbb{R}}\left(\left\{|\mathbf{x}|^{\alpha} \mid \alpha \in \mathcal{A}\right\} \cup\left\{\mathbf{x}^{\beta} \mid \beta \in \mathcal{B}\right\}\right)
$$

denote the space of all functions of the form (2) with given sets of exponents. This is a vector space of dimension $\operatorname{dim} \mathbb{R}[\mathcal{A}, \mathcal{B}]=|\mathcal{A}|+|\mathcal{B}|$.

Remark 2.1. (1) If $\mathcal{A} \subseteq(2 \mathbb{N})^{n}$, then $\mathbb{R}[\mathcal{A}, \mathcal{B}]$ is exactly the space of polynomials with exponent vectors in $\mathcal{A} \cup \mathcal{B}$. For this reason, we sometimes refer to elements of $\mathcal{A}$ as even exponents and to elements of $\mathcal{B}$ as odd exponents. 
(2) If $\mathcal{B}=\emptyset$, then $\mathbb{R}[\mathcal{A}, \mathcal{B}]$ can be identified with the space of signomials, i.e., functions of the form

$$
\mathbf{y} \mapsto \sum_{\alpha \in \mathcal{A}} c_{\alpha} \exp \left(\alpha^{T} \mathbf{y}\right)
$$

via the identification $\left|x_{i}\right|=\exp \left(y_{i}\right)$.

(3) It is no restriction to exclude sets in $(2 \mathbb{N})^{n}$ from $\mathcal{B}$, since for exponents $\beta \in(2 \mathbb{N})^{n}$, we have $|\mathbf{x}|^{\beta}=\mathbf{x}^{\beta}$.

(4) $\mathcal{A}$ and $\mathcal{B}$ are not necessarily disjoint (cf. Example 2.9 below).

We study the non-negativity of functions in $\mathbb{R}[\mathcal{A}, \mathcal{B}]$ using the following building blocks:

Definition 2.2. Let $f=\sum_{\alpha \in \mathcal{A}} c_{\alpha}|\mathbf{x}|^{\alpha}+\sum_{\beta \in \mathcal{B}} d_{\beta} \mathbf{x}^{\beta}$. We say that $f$ is

(1) an even $A G$ function if at most one of the $c_{\alpha}$ is negative and all the $d_{\beta}$ are zero; and

(2) an odd $A G$ function if all the $c_{\alpha}$ are non-negative and at most one of the $d_{\beta}$ is nonzero. $f$ is called an $A G$ function (arithmetic-geometric mean function) if $f$ is an even AG function or an odd AG function.

Note that non-negative even AG functions correspond exactly to the AGE functions (arithmeticgeometric exponentials) studied in [4] and [21].

We arrive at the central definition of this section.

Definition $2.3\left(\mathcal{S}\right.$-cone). Let $\emptyset \neq \mathcal{A} \subseteq \mathbb{R}^{n}, \mathcal{B} \subseteq \mathbb{N}^{n} \backslash(2 \mathbb{N})^{n}$ be finite sets. The $\mathcal{S}$-cone $C_{\mathcal{S}}(\mathcal{A}, \mathcal{B})$ is defined as

$$
C_{\mathcal{S}}(\mathcal{A}, \mathcal{B}):=\operatorname{cone}(f \in \mathbb{R}[\mathcal{A}, \mathcal{B}] \mid f \text { is a non-negative } \mathrm{AG} \text { function }),
$$

where cone denotes the conic hull.

\section{Remark 2.4.}

(1) If $\mathcal{B}=\emptyset$, then the $\mathcal{S}$-cone can be identified with the SAGE cone using the substitution in Remark 2.1(2). Formally, for finite $\mathcal{A} \subseteq \mathbb{R}^{n}, \mathcal{A}^{\prime} \subsetneq \mathcal{A}$ and $\beta \in \mathcal{A} \backslash \mathcal{A}^{\prime}$, we set

$$
C_{\mathrm{SAGE}}(\mathcal{A})=\sum_{\beta \in \mathcal{A}} C_{\mathrm{AGE}}(\mathcal{A} \backslash\{\beta\}, \beta),
$$

where for $\mathcal{A}^{\prime}:=\mathcal{A} \backslash\{\beta\}$

$$
C_{\mathrm{AGE}}\left(\mathcal{A}^{\prime}, \beta\right)=\left\{c \in \mathbb{R}^{\mathcal{A}}: c_{\alpha} \geq 0 \text { for } \alpha \in \mathcal{A}^{\prime}, \sum_{\alpha \in \mathcal{A}^{\prime}} c_{\alpha} \exp \left(\alpha^{T} x\right)+c_{\beta} \exp \left(\beta^{T} x\right) \geq 0 \text { on } \mathbb{R}^{n}\right\} \text {. }
$$

(2) If $\mathcal{A} \subseteq(2 \mathbb{N})^{n}$, then $C_{\mathcal{S}}(\mathcal{A}, \mathcal{B})$ is the cone of SONC polynomials supported on $\mathcal{A} \cup \mathcal{B}$ from $[13,3]$. In those papers, the SONC cone is defined in terms of circuit polynomials (see Remark 3.3). The equivalence of the definitions was established in [21] and also follows from our more general result in Proposition 4.1.

(3) An example where the cone $C_{\mathcal{S}}(\mathcal{A}, \mathcal{B})$ is different from both the SAGE cone and the SONC cone is given by $\mathcal{A}=\{1,4\}$ and $\mathcal{B}=\{3\}$. 
For a non-empty finite set $\mathcal{A} \subseteq \mathbb{R}^{n}$ and $\beta \in \mathbb{N}^{n} \backslash(2 \mathbb{N})^{n}$ let

$$
P_{\mathcal{A}, \beta}^{\text {odd }}:=\left\{\left.f\left|f=\sum_{\alpha \in \mathcal{A}} c_{\alpha}\right| \mathbf{x}\right|^{\alpha}+d \mathbf{x}^{\beta}, f(\mathbf{x}) \geq 0 \forall \mathbf{x} \in \mathbb{R}^{n}, c \in \mathbb{R}_{+}^{\mathcal{A}}, d \in \mathbb{R}\right\}
$$

be the cone of non-negative odd AG functions supported on $(\mathcal{A}, \beta)$, and similarly for $\beta \in \mathbb{R}^{n} \backslash \mathcal{A}$ let

$$
P_{\mathcal{A}, \beta}^{\text {even }}:=\left\{\left.f\left|f=\sum_{\alpha \in \mathcal{A}} c_{\alpha}\right| \mathbf{x}\right|^{\alpha}+d|\mathbf{x}|^{\beta}, f(\mathbf{x}) \geq 0 \forall \mathbf{x} \in \mathbb{R}^{n}, c \in \mathbb{R}_{+}^{\mathcal{A}}, d \in \mathbb{R}\right\}
$$

be the cone of non-negative even $\mathrm{AG}$ functions supported on $(\mathcal{A}, \beta)$. Note that, by definition,

$$
C_{\mathcal{S}}(\mathcal{A}, \mathcal{B})=\sum_{\alpha \in \mathcal{A}} P_{\mathcal{A} \backslash\{\alpha\}, \alpha}^{\mathrm{even}}+\sum_{\beta \in \mathcal{B}} P_{\mathcal{A}, \beta}^{\text {odd }}
$$

As pointed out by a referee, (3) implies the following alternative representation of the $\mathcal{S}$-cone.

Proposition 2.5. Let $\emptyset \neq \mathcal{A} \subseteq \mathbb{R}^{n}, \mathcal{B} \subseteq \mathbb{N}^{n} \backslash(2 \mathbb{N})^{n}$ be finite and $\mathbf{e}_{\alpha}$ denote the unit vector in $\mathbb{R}^{\mathcal{A} \cup \mathcal{B}}$ indexed with $\alpha \in \mathcal{A} \cup \mathcal{B}$. Then,

$$
\begin{aligned}
C_{\mathcal{S}}(\mathcal{A}, \mathcal{B})= & \left\{\sum_{\alpha \in \mathcal{A}} c_{\alpha}|x|^{\alpha}+\sum_{\beta \in \mathcal{B}} d_{\beta} x^{\beta} \in \mathbb{R}[\mathcal{A}, \mathcal{B}]: \sum_{\alpha \in \mathcal{A}} c_{\alpha} \cdot \mathbf{e}_{\alpha}-\sum_{\beta \in \mathcal{B}}\left|d_{\beta}\right| \cdot \mathbf{e}_{\beta} \in C_{\mathrm{SAGE}}(\mathcal{A} \cup \mathcal{B})\right\} \\
= & \left\{\sum_{\alpha \in \mathcal{A}} c_{\alpha}|x|^{\alpha}+\sum_{\beta \in \mathcal{B}} d_{\beta} x^{\beta} \in \mathbb{R}[\mathcal{A}, \mathcal{B}]:\right. \\
& \left.\exists t \in \mathbb{R}^{\mathcal{B}}, \sum_{\alpha \in \mathcal{A}} c_{\alpha} \cdot \mathbf{e}_{\alpha}+\sum_{\beta \in \mathcal{B}} t_{\beta} \cdot \mathbf{e}_{\alpha} \in C_{\mathrm{SAGE}}(\mathcal{A} \cup \mathcal{B}), t_{\beta} \leq-\left|d_{\beta}\right| \text { for all } \beta \in \mathcal{B}\right\} .
\end{aligned}
$$

In the case $\mathcal{A} \cap \mathcal{B}=\emptyset$, we can shortly write $\sum_{\alpha \in \mathcal{A}} c_{\alpha} \cdot \mathbf{e}_{\alpha}-\sum_{\beta \in \mathcal{B}}\left|d_{\beta}\right| \cdot \mathbf{e}_{\beta}=(c,-|d|)$, where $|d|$ denotes the component-wise absolute value. If there exists some $\beta \in \mathcal{A} \cap \mathcal{B}$, then the corresponding coefficient in the SAGE cone $c_{\beta}-\left|d_{\beta}\right|$ appears only once in the set $\mathbb{R}^{\mathcal{A} \cup \mathcal{B}}$. However, by slight abuse of notation, we also write $\sum_{\alpha \in \mathcal{A}} c_{\alpha} \cdot \mathbf{e}_{\alpha}-\sum_{\beta \in \mathcal{B}}\left|d_{\beta}\right| \cdot \mathbf{e}_{\beta}$ shortly as $(c,-|d|)$.

Proof. If $f=\sum_{\alpha \in \mathcal{A}} c_{\alpha}|x|^{\alpha}+\sum_{\beta \in \mathcal{B}} d_{\beta} x^{\beta} \in C_{\mathcal{S}}(\mathcal{A}, \mathcal{B})$, then (3) gives a decomposition

$$
f=\sum_{\alpha \in \mathcal{A}} f_{\alpha}^{\text {even }}+\sum_{\beta \in \mathcal{B}} f_{\beta}^{\text {odd }}
$$

with $f_{\alpha}^{\text {even }} \in P_{\mathcal{A} \backslash\{\alpha\}, \alpha}^{\text {even }}$ for all $\alpha \in \mathcal{A}$ and $f_{\beta}^{\text {odd }}=\sum_{\alpha \in \mathcal{A}} c_{\alpha}^{(\beta)}|x|^{\alpha}+d_{\beta} x^{\beta} \in P_{\mathcal{A}, \beta}^{\text {odd }}$ for every $\beta \in \mathcal{B}$. Defining the functions

$$
\begin{aligned}
\tilde{f}_{\alpha}^{\text {even }} & =f_{\alpha}^{\text {even }} \text { for all } \alpha \in \mathcal{A} \\
\text { and } \tilde{f}_{\beta}^{\text {even }} & =\sum_{\alpha \in \mathcal{A}} c_{\alpha}^{(\beta)}|x|^{\alpha}-\left|d_{\beta}\right||x|^{\beta}=f_{\beta}^{\text {odd }}-d_{\beta} x^{\beta}-\left|d_{\beta}\right||x|^{\beta} \text { for all } \beta \in \mathcal{B},
\end{aligned}
$$


symmetry implies $\tilde{f}_{\beta}^{\text {even }} \in P_{\mathcal{A}, \beta}^{\text {even }}$ and hence, $\tilde{f}=\sum_{\alpha \in \mathcal{A}} \tilde{f}_{\alpha}^{\text {even }}+\sum_{\beta \in \mathcal{B}} \tilde{f}_{\beta}^{\text {even }} \in C_{\mathcal{S}}(\mathcal{A} \cup \mathcal{B}, \emptyset)$. Remark 2.4(1) then shows that the coefficient vector of $\tilde{f}$ is contained in $C_{\mathrm{SAGE}}(\mathcal{A} \cup \mathcal{B})$.

The converse direction of the first equation follows immediately with the substitution in Remark 2.1(2).

The second equation, which exhibits the convexity of the $\mathcal{S}$-cone, is an immediate consequence of the first one.

In our definition of the $\mathcal{S}$-cone, we exclude sums of non-negative AG functions with support $\mathcal{A} \cup \mathcal{B}$ for $\mathcal{A} \subseteq \mathbb{R}^{n}, \mathcal{B} \subseteq \mathbb{N}^{n} \backslash(2 \mathbb{N})^{n}$, where the corresponding AG functions have bigger support than $\mathcal{A} \cup \mathcal{B}$. This could happen, for example, if two summands cancel in the sum. For a better understanding of the problem, we have a look at the following example.

Example 2.6. Let $\mathcal{A}:=\left\{\frac{1}{3}, \frac{7}{3}\right\}, \mathcal{B}:=\{1\}$. Consider the two non-negative AG functions

$$
\begin{aligned}
& f_{1}:=|x|^{\frac{1}{3}}+x+x^{2}, \\
& f_{2}:=|x|^{\frac{1}{3}}-x^{2}+|x|^{\frac{7}{3}},
\end{aligned}
$$

whose support is not contained in $\mathcal{A} \cup \mathcal{B}$. But the sum

$$
f:=f_{1}+f_{2}=2|x|^{\frac{1}{3}}+x+|x|^{\frac{7}{3}}
$$

is itself a non-negative $\mathrm{AG}$ function, whose support is contained in $\mathcal{A} \cup \mathcal{B}$.

In fact, this restriction is not really a restriction. The following proposition states that every sum $f$ of non-negative AG functions whose support is bigger than the support of the sum can be decomposed into a sum of non-negative AG functions whose supports are contained in the support of $f$.

For the SAGE case, this was already proven in [21, Theorem 2] and for the SONC case this follows from the more detailed result of [26].

Proposition 2.7. Let $\emptyset \neq \mathcal{A} \subseteq \mathbb{R}^{n}, \mathcal{B} \subseteq \mathbb{N}^{n} \backslash(2 \mathbb{N})^{n}$ be finite sets and $f \in \mathbb{R}[\mathcal{A}, \mathcal{B}]$. If $f \in C_{\mathcal{S}}\left(\mathcal{A}^{\prime}, \mathcal{B}^{\prime}\right)$ for some $\mathcal{A}^{\prime} \supseteq \mathcal{A}, \mathbb{N}^{n} \backslash(2 \mathbb{N})^{n} \supseteq \mathcal{B}^{\prime} \supseteq \mathcal{B}$, then $f \in C_{\mathcal{S}}(\mathcal{A}, \mathcal{B})$ as well. Equivalently, it holds that

$$
C_{\mathcal{S}}(\mathcal{A}, \mathcal{B})=C_{\mathcal{S}}\left(\mathcal{A}^{\prime}, \mathcal{B}^{\prime}\right) \cap \mathbb{R}[\mathcal{A}, \mathcal{B}]
$$

Proof. By Proposition 2.5,

$$
C_{\mathcal{S}}(\mathcal{A}, \mathcal{B})=\left\{\sum_{\alpha \in \mathcal{A}} c_{\alpha}|x|^{\alpha}+\sum_{\beta \in \mathcal{B}} d_{\beta} x^{\beta} \in \mathbb{R}[\mathcal{A}, \mathcal{B}]: \sum_{\alpha \in \mathcal{A}} c_{\alpha} \cdot \mathbf{e}_{\alpha}-\sum_{\beta \in \mathcal{B}}\left|d_{\beta}\right| \cdot \mathbf{e}_{\beta} \in C_{\mathrm{SAGE}}(\mathcal{A} \cup \mathcal{B})\right\},
$$

with $\mathbf{e}_{\alpha}$ denoting the unit vector with respect to $\alpha$ for $\alpha \in \mathcal{A}$, resp. $\mathcal{B}$. Let $f \in C_{\mathcal{S}}\left(\mathcal{A}^{\prime}, \mathcal{B}^{\prime}\right) \cap$ $\mathbb{R}[\mathcal{A}, \mathcal{B}]$ with coefficient vector $(c, d)$ and hence $(c,-|d|) \in C_{\mathrm{SAGE}}\left(\mathcal{A}^{\prime} \cup \mathcal{B}^{\prime}\right) \cap \mathbb{R}^{\mathcal{A} \cup \mathcal{B}}$ where the absolute value is component-wise. The already mentioned statement for the SAGE-case ([21], Theorem 2) states that $C_{\mathrm{SAGE}}(\mathcal{A} \cup \mathcal{B})=C_{\mathrm{SAGE}}\left(\mathcal{A}^{\prime} \cup \mathcal{B}^{\prime}\right) \cap \mathbb{R}^{\mathcal{A} \cup \mathcal{B}}$. Hence, $(c,-|d|) \in C_{\mathrm{SAGE}}(\mathcal{A} \cup \mathcal{B})$ 
as well as

$$
\begin{aligned}
f & \in\left\{\sum_{\alpha \in \mathcal{A}} c_{\alpha}|x|^{\alpha}+\sum_{\beta \in \mathcal{B}} d_{\beta} x^{\beta} \in \mathbb{R}[\mathcal{A}, \mathcal{B}]: \sum_{\alpha \in \mathcal{A}} c_{\alpha} \cdot \mathbf{e}_{\alpha}-\sum_{\beta \in \mathcal{B}}\left|d_{\beta}\right| \cdot \mathbf{e}_{\beta} \in C_{\mathrm{SAGE}}(\mathcal{A} \cup \mathcal{B})\right\} \\
& =C_{\mathcal{S}}(\mathcal{A}, \mathcal{B}),
\end{aligned}
$$

again by Proposition 2.5. The other inclusion is obvious.

Our next result characterizes non-negative AG functions. It is a slight generalization of [5, Lemma 2.2] to the setting of AG functions. The following notation is useful to state the theorem:

Notation. For a non-empty finite set $\mathcal{A} \subseteq \mathbb{R}^{n}$ and $\beta \in \mathbb{R}^{n}$, let $\Lambda(\mathcal{A}, \beta)$ be the polytope

$$
\Lambda(\mathcal{A}, \beta):=\left\{\lambda \in \mathbb{R}_{+}^{\mathcal{A}} \mid \sum_{\alpha \in \mathcal{A}} \lambda_{\alpha} \alpha=\beta, \sum_{\alpha \in \mathcal{A}} \lambda_{\alpha}=1\right\} .
$$

Note that $\Lambda(\mathcal{A}, \beta) \neq \emptyset$ if and only if $\beta$ is contained in the convex hull of $\mathcal{A}$. In the special case that $\mathcal{A}$ is affinely independent, $\Lambda(\mathcal{A}, \beta)$ consists of a single element, which we denote by $\lambda(\mathcal{A}, \beta)$.

Let $\mathcal{A} \subseteq \mathbb{R}^{n}$ be a non-empty finite set. We denote by $D: \mathbb{R}_{>0}^{\mathcal{A}} \times \mathbb{R}_{>0}^{\mathcal{A}} \rightarrow \mathbb{R}$,

$$
D(\nu, \gamma)=\sum_{\alpha \in \mathcal{A}} \nu_{\alpha} \ln \left(\frac{\nu_{\alpha}}{\gamma_{\alpha}}\right), \quad \nu, \gamma \in \mathbb{R}_{>0}^{\mathcal{A}}
$$

the relative entropy function. It can be extended to $\mathbb{R}_{+}^{\mathcal{A}} \times \mathbb{R}_{+}^{\mathcal{A}} \rightarrow \mathbb{R} \cup\{\infty\}$ using the usual conventions $0 \cdot \ln \frac{0}{y}=0$ for $y \geq 0$ and $y \cdot \ln \frac{y}{0}=\infty$ for $y>0$.

Theorem 2.8. Let $\mathcal{A} \subseteq \mathbb{R}^{n}$ be a non-empty finite set and $f$ be an $A G$ function of the form

$$
f=\sum_{\alpha \in \mathcal{A}} c_{\alpha}|\mathbf{x}|^{\alpha}+ \begin{cases}d|\mathbf{x}|^{\beta} \text { with } \beta \in \mathbb{R}^{n} \backslash \mathcal{A} & \text { if } f \text { is even, } \\ d \mathbf{x}^{\beta} \text { with } \beta \in \mathbb{N}^{n} \backslash(2 \mathbb{N})^{n} & \text { if } f \text { is odd, }\end{cases}
$$

where $c_{\alpha} \geq 0$ for all $\alpha \in \mathcal{A}$ and $d \in \mathbb{R}$. Then the following statements are equivalent:

(1) $f(\mathbf{x}) \geq 0$ for all $\mathbf{x} \in \mathbb{R}^{n}$.

(2) There exists a $\nu \in \mathbb{R}_{+}^{\mathcal{A}}$ such that $\sum_{\alpha \in \mathcal{A}} \nu_{\alpha} \alpha=\left(\sum_{\alpha \in \mathcal{A}} \nu_{\alpha}\right) \beta$ and

$$
D(\nu, e \cdot c) \leq \begin{cases}d & \text { if } f \text { even } \\ -|d| & \text { if } f \text { odd }\end{cases}
$$

(3) There exists a $\lambda \in \Lambda(\mathcal{A}, \beta)$ such that

$$
\prod_{\alpha \in \mathcal{A}}\left(\frac{c_{\alpha}}{\lambda_{\alpha}}\right)^{\lambda_{\alpha}} \geq \begin{cases}-d & \text { if } f \text { even } \\ |d| & \text { if } f \text { odd } .\end{cases}
$$

A vector $\lambda \in \Lambda(\mathcal{A}, \beta)$ as in this theorem is called an $A G$ witness. 
Proof of Theorem 2.8. Before we prove this theorem, observe that for the AGE cone $C_{\mathrm{AGE}}(\mathcal{A}, \beta)$, defined in Remark 2.4, we have $(c, d) \in C_{\mathrm{AGE}}(\mathcal{A}, \beta)$ if and only if there exists $\nu \in \mathbb{R}_{+}^{\mathcal{A}}$ such that $\sum_{\alpha \in \mathcal{A}} \nu_{\alpha} \alpha=\left(\sum_{\alpha \in \mathcal{A}} \nu_{\alpha}\right) \beta$ and

$$
D(\nu, e \cdot c) \leq d
$$

(compare [4], Section 2.1). If $f$ is an even AG-function, this is exactly the equivalence $(1) \Leftrightarrow(2)$ due to Remark 2.4. If $f$ is an odd AG-function, the equivalence follows from Proposition 2.5 and the mentioned observation.

For the implication $(2) \Longrightarrow(3)$, set $\lambda:=\left(\sum_{\alpha \in \mathcal{A}} \nu_{\alpha}\right)^{-1} \nu$. It is clear from the properties of $\nu$ that $\lambda \in \Lambda(\mathcal{A}, \beta)$. The discussion in $[4$, p. 1151] shows that

$$
\prod_{\alpha \in \mathcal{A}}\left(\frac{c_{\alpha}}{\lambda_{\alpha}}\right)^{\lambda_{\alpha}} \geq-D(\nu, e \cdot c)
$$

and thus this $\lambda$ has the desired properties. The implication $(3) \Longrightarrow(1)$ is a direct consequence of the weighted arithmetic-geometric mean inequality:

$$
\sum_{\alpha \in \mathcal{A}} c_{\alpha}|x|^{\alpha} \stackrel{\text { AM/GM-inequality }}{\geq} \prod_{\alpha \in \mathcal{A}}\left(\frac{c_{\alpha}}{\lambda_{\alpha}}|x|^{\alpha}\right)^{\lambda_{\alpha}}=\prod_{\alpha \in \mathcal{A}}\left(\frac{c_{\alpha}}{\lambda_{\alpha}}\right)^{\lambda_{\alpha}}|x|^{\beta} .
$$

Using (3), we obtain

$$
\sum_{\alpha \in \mathcal{A}} c_{\alpha}|x|^{\alpha}+\left\{\begin{array}{l}
d|x|^{\beta} \\
d x^{\beta}
\end{array} \geq|x|^{\beta}\left\{\begin{array}{l}
-d+d \\
|d|-\operatorname{sgn}(x) \cdot d
\end{array} \geq 0 .\right.\right.
$$

As we already know that $(1) \Leftrightarrow(2)$, we obtain the desired statement.

Example 2.9. Let $\mathcal{A}=\mathcal{B}=\{1\} \subseteq \mathbb{N}$. A typical AG function with this support is

$$
g(x)=c_{1}|x|+c_{2} x
$$

Since the equality condition in statement (2) of Theorem 2.8 is trivially satisfied, we have $g(x) \geq 0$ for all $x \in \mathbb{R}$ if and only if there exists a $\nu \in \mathbb{R}_{+}$with

$$
\nu \ln \left(\frac{\nu}{e c_{1}}\right) \leq-\left|c_{2}\right|
$$

If $\nu \geq 0$, the latter condition can be simplified to $\left|c_{2}\right| \leq c_{1}$. For the case $\nu=0$, this is clear from our setting $0 \cdot \ln 0=0$, and to see it for $\nu>0$, rewrite (5) as

$$
c_{1}\left(\frac{\nu}{c_{1}}\right) \ln \left(\frac{\nu}{e c_{1}}\right) \leq-\left|c_{2}\right| \text {. }
$$

Since the function $x \ln \left(\frac{x}{e}\right)$ attains its minimum at $x=1$ (which means $x \ln \left(\frac{x}{e}\right) \geq-1$ ), we obtain the claimed result. It is in particular the one of statement (3) in Theorem 2.8.

For later use, we note that our cones of interest are closed:

Proposition 2.10. The cones $P_{\mathcal{A}, \beta}^{\text {odd }}, P_{\mathcal{A}, \beta}^{\text {even }}$ and $C_{\mathcal{S}}(\mathcal{A}, \mathcal{B})$ are closed pointed convex cones. 
Proof. It is clear that all three cones are pointed, since the only non-negative function $f$ where $-f$ is non-negative as well is the zero function. The cones $P_{\mathcal{A}, \beta}^{\text {odd }}$ and $P_{\mathcal{A}, \beta}^{\text {even }}$ are defined as (infinite) intersections of closed halfspaces, and thus they are closed. Finally, since finite sums of closed pointed convex cones are again closed, the cone $C_{\mathcal{S}}(\mathcal{A}, \mathcal{B})$ is closed as well.

\section{Circuits and the dual of the $\mathcal{S}$-Cone}

In this section, we introduce circuit functions and provide several characterizations of the dual $\mathcal{S}$-cone (see Theorem 3.5).

We can identify the dual space of $\mathbb{R}[\mathcal{A}, \mathcal{B}]$ with $\mathbb{R}^{(\mathcal{A}, \mathcal{B})}:=\mathbb{R}^{\mathcal{A}} \times \mathbb{R}^{\mathcal{B}}$. For $f \in \mathbb{R}[\mathcal{A}, \mathcal{B}]$ with coefficients $\left(c_{\alpha}\right)_{\alpha \in \mathcal{A}},\left(d_{\beta}\right)_{\beta \in \mathcal{B}}$ and an element $(v, w) \in \mathbb{R}^{(\mathcal{A}, \mathcal{B})}$, we consider the natural pairing

$$
(v, w)(f)=\sum_{\alpha \in \mathcal{A}} v_{\alpha} c_{\alpha}+\sum_{\beta \in \mathcal{B}} w_{\beta} d_{\beta} .
$$

Using this notation, the dual cone $C_{\mathcal{S}}(\mathcal{A}, \mathcal{B})^{*}$ is defined as

$$
C_{\mathcal{S}}(\mathcal{A}, \mathcal{B})^{*}=\left\{(v, w) \in \mathbb{R}^{(\mathcal{A}, \mathcal{B})} \mid(v, w)(f) \geq 0 \text { for all } f \in C_{\mathcal{S}}(\mathcal{A}, \mathcal{B})\right\} .
$$

Now we consider the representation of AG functions in terms of circuit functions. Here, relint and conv denote the relative interior and the convex hull of a set.

Definition 3.1. A circuit is a pair $(A, \beta)$, where $A \subseteq \mathbb{R}^{n}$ is affinely independent and $\beta \in$ relint $\operatorname{conv}(A)$. For finite sets $\mathcal{A}, \mathcal{B} \subseteq \mathbb{R}^{n}$, let

$$
I(\mathcal{A}, \mathcal{B}):=\{(A, \beta) \text { circuit } \mid A \subseteq \mathcal{A}, \beta \in \mathcal{B}\}
$$

denote the set of all circuits on $\mathcal{A}, \mathcal{B}$. In particular, for $\mathcal{A} \subseteq \mathbb{R}^{n}, \mathcal{B} \subseteq \mathbb{N}^{n} \backslash(2 \mathbb{N})^{n}$ we call $I(\mathcal{A}, \mathcal{A})$ the set of all even circuits and $I(\mathcal{A}, \mathcal{B})$ the set of all odd circuits.

Definition 3.2. Let $(A, \beta)$ be a circuit.

(1) An even circuit function supported on $(A, \beta)$ is an AG function of the form

$$
f=\sum_{\alpha \in A} c_{\alpha}|\mathbf{x}|^{\alpha}+d|\mathbf{x}|^{\beta}
$$

with $c_{\alpha}>0$ for all $\alpha \in A$ and $d \in \mathbb{R}$.

(2) For $\beta \in \mathbb{N}^{n} \backslash(2 \mathbb{N})^{n}$, an odd circuit function supported on $(A, \beta)$ is an AG function of the form

$$
f=\sum_{\alpha \in A} c_{\alpha}|\mathbf{x}|^{\alpha}+d \mathbf{x}^{\beta}
$$

with $c_{\alpha}>0$ for all $\alpha \in A$ and $d \in \mathbb{R}$.

We call $\beta$ the inner exponent of $f$ and the other exponents are the outer exponents.

Remark 3.3. (1) In case of a circuit, the vector $\lambda \in \Lambda(\mathcal{A}, \beta)$ in Theorem 2.8 is unique, and thus the non-negativity of $f$ can be expressed in terms of the circuit number

$$
\Theta_{f}=\prod_{\lambda_{\alpha} \neq 0}\left(\frac{c_{\alpha}}{\lambda_{\alpha}}\right)^{\lambda_{\alpha}},
$$

which was introduced in [13]. 
(2) Iliman and de Wolff also introduced the notion of circuit polynomials in [13]. Every circuit polynomial is an even circuit function if the inner exponent is even and an odd circuit function if the inner exponent is odd. With this, circuit polynomials form a special case of circuit functions.

Next, we introduce reduced circuits, which will be used in Section 4.2 to determine the extreme rays of the $\mathcal{S}$-cone.

Definition 3.4. For a circuit $(A, \beta)$ let

$$
\begin{aligned}
& r_{\mathrm{e}}(A, \beta):=|(\operatorname{conv}(A) \backslash(A \cup\{\beta\})) \cap \mathcal{A}| \text { and } \\
& r_{\mathrm{o}}(A, \beta):=|(\operatorname{conv}(A) \backslash A) \cap \mathcal{A}| .
\end{aligned}
$$

An even circuit $(A, \beta)$ is called reduced if $r_{\mathrm{e}}(A, \beta)=0$ and an odd circuit $(A, \beta)$ is called reduced if $r_{\mathrm{o}}(A, \beta)=0$.

In other words, reduced circuits contain no elements of $\mathcal{A}$ in their convex hull except those which are trivially there. Note that for $\beta \in \mathcal{A} \cap \mathcal{B}$, it is possible that a circuit is reduced as an even circuit, but not reduced as an odd circuit. See Example 4.5 below.

We can now provide the following characterization of the dual $\mathcal{S}$-cone $C_{\mathcal{S}}(\mathcal{A}, \mathcal{B})^{*}$. Here, recall the definition of $\Lambda(\mathcal{A}, \beta)$ from $(4)$ and that $\lambda(\mathcal{A}, \beta)$ denotes the single element of $\Lambda(\mathcal{A}, \beta)$ in the case of a circuit. We use the convention that $0 \ln (0)=0$ and $\ln (0)=-\infty$.

Theorem 3.5. Let $\emptyset \neq \mathcal{A} \subseteq \mathbb{R}^{n}$ and $\mathcal{B} \subseteq \mathbb{N}^{n} \backslash(2 \mathbb{N})^{n}$ be finite sets and let $(v, w) \in \mathbb{R}^{(\mathcal{A}, \mathcal{B})}$.

(1) If $(v, w) \in C_{\mathcal{S}}(\mathcal{A}, \mathcal{B})^{*}$, then $v_{\alpha} \geq 0$ for all $\alpha \in \mathcal{A}$.

(2) If the condition of part (1) is satisfied, then the following are equivalent:

(a) $(v, w)$ lies in the dual cone $C_{\mathcal{S}}(\mathcal{A}, \mathcal{B})^{*}$.

(b) For all $\beta \in \mathcal{A}$ (respectively $\beta \in \mathcal{B}$ ) and all $\lambda \in \Lambda(\mathcal{A}, \beta)$, it holds that

$$
\left.\ln \left|v_{\beta}\right| \leq \sum_{\alpha \in \mathcal{A}} \lambda_{\alpha} \ln \left(v_{\alpha}\right) \quad \text { (respectively } \ln \left|w_{\beta}\right| \leq \sum_{\alpha \in \mathcal{A}} \lambda_{\alpha} \ln \left(v_{\alpha}\right)\right) .
$$

(c) For every even circuit $(A, \beta) \in I(\mathcal{A}, \mathcal{A})$ (respectively odd circuit $(A, \beta) \in I(\mathcal{A}, \mathcal{B})$ ) and $\lambda=\lambda(A, \beta)$, it holds that

$$
\left.\ln \left|v_{\beta}\right| \leq \sum_{\alpha \in A} \lambda_{\alpha} \ln \left(v_{\alpha}\right) \quad \text { (respectively } \ln \left|w_{\beta}\right| \leq \sum_{\alpha \in A} \lambda_{\alpha} \ln \left(v_{\alpha}\right)\right) .
$$

(d) For every reduced even circuit $(A, \beta) \in I(\mathcal{A}, \mathcal{A})$ (respectively reduced odd circuit $(A, \beta) \in$ $I(\mathcal{A}, \mathcal{B})$ ) and $\lambda=\lambda(A, \beta)$, it holds that

$$
\left.\ln \left|v_{\beta}\right| \leq \sum_{\alpha \in A} \lambda_{\alpha} \ln \left(v_{\alpha}\right) \quad \text { (respectively } \ln \left|w_{\beta}\right| \leq \sum_{\alpha \in A} \lambda_{\alpha} \ln \left(v_{\alpha}\right)\right) .
$$

Before we prove Theorem 3.5 we consider the duals of the sub-cones $P_{\mathcal{A}, \beta}^{\text {odd }}$ and $P_{\mathcal{A}, \beta}^{\text {even }}$ of $C_{\mathcal{S}}(\mathcal{A}, \mathcal{B})$.

Lemma 3.6. Let $\mathcal{A} \subseteq \mathbb{R}^{n}$ be a non-empty finite set.

(1) For $\beta \in \mathbb{N}^{n} \backslash(2 \mathbb{N})^{n}$, the dual cone of $P_{\mathcal{A}, \beta}^{\text {odd }}$ consists of those $(v, w) \in \mathbb{R}^{(\mathcal{A},\{\beta\})}$ where (a) $v_{\alpha} \geq 0$ for all $\alpha \in \mathcal{A}$, and 
(b) $\ln \left|w_{\beta}\right| \leq \sum_{\alpha \in \mathcal{A}} \lambda_{\alpha} \ln \left(v_{\alpha}\right)$ for all $\lambda \in \Lambda(\mathcal{A}, \beta)$.

(2) For $\beta \in \mathbb{R}^{n} \backslash \mathcal{A}$, the dual cone of $P_{\mathcal{A}, \beta}^{\text {even }}$ consists of those $(v, w) \in \mathbb{R}^{(\mathcal{A},\{\beta\})}$ satisfying (a), (b) and in addition

(c) $w_{\beta} \geq 0$.

Proof. We prove the even and odd case simultaneously. Let $(v, w) \in\left(P_{\mathcal{A}, \beta}^{\text {odd }}\right)^{*}$ or $(v, w) \in\left(P_{\mathcal{A}, \beta}^{\text {even }}\right)^{*}$. First we show that it satisfies the claimed conditions.

(a) and (c): For every $\alpha \in \mathcal{A}$, it holds that $|\mathbf{x}|^{\alpha} \in P_{\mathcal{A}, \beta}^{\text {odd }}$ resp. $|\mathbf{x}|^{\alpha} \in P_{\mathcal{A}, \beta}^{\text {even }}$ and thus $0 \leq$ $(v, w)\left(|\mathbf{x}|^{\alpha}\right)=v_{\alpha}$, as claimed. In the even case, we also have that $|\mathbf{x}|^{\beta} \in P_{\mathcal{A}, \beta}^{\text {even }}$ and thus by the same argument $(c)$ holds.

(b): Fix a $\lambda \in \Lambda(\mathcal{A}, \beta)$. First assume that $v_{\alpha} \neq 0$ for all $\alpha \in \mathcal{A}$. Then

$$
f:=\sum_{\alpha \in \mathcal{A}}\left(\prod_{\alpha^{\prime} \in \mathcal{A}} v_{\alpha^{\prime}}^{\lambda_{\alpha^{\prime}}}\right) \frac{\lambda_{\alpha}}{v_{\alpha}}|\mathbf{x}|^{\alpha}- \begin{cases}|\mathbf{x}|^{\beta} & \text { in the even case } \\ \operatorname{sgn}\left(w_{\beta}\right) \mathbf{x}^{\beta} & \text { in the odd case }\end{cases}
$$

is an (even or odd) AG function and a straightforward computation shows that $f$ satisfies the condition (3) of Theorem 2.8 (with the given $\lambda$ ), hence $f$ is non-negative. Thus,

$$
0 \leq(v, w)(f)= \begin{cases}\prod_{\alpha \in \mathcal{A}} v_{\alpha}^{\lambda_{\alpha}}-w_{\beta} & \text { in the even case } \\ \prod_{\alpha \in \mathcal{A}} v_{\alpha}^{\lambda_{\alpha}}-\operatorname{sgn}\left(w_{\beta}\right) w_{\beta} & \text { in the odd case }\end{cases}
$$

which is equivalent to property (b). Since the mapping (6) is continuous in $(v, w)$, the statements also hold if $v_{\alpha}=0$ for some $\alpha \in \mathcal{A}$.

For the converse implication, assume that $v$ satisfies conditions $(a),(b)$, and in the even case also (c).

We need to show that every non-negative AG function $f=\sum_{\alpha \in \mathcal{A}} c_{\alpha}|\mathbf{x}|^{\alpha}+d \mathbf{x}^{\beta}$ resp. $f=$ $\sum_{\alpha \in \mathcal{A}} c_{\alpha}|\mathbf{x}|^{\alpha}+d|\mathbf{x}|^{\beta}$ satisfies $(v, w)(f) \geq 0$. Let $\lambda \in \Lambda(\mathcal{A}, \beta)$ be an AG witness for $f$ as in Theorem 2.8. Observe that

$$
\sum_{\alpha \in \mathcal{A}} v_{\alpha} c_{\alpha}=\sum_{\alpha \in \mathcal{A}} \lambda_{\alpha}\left(\frac{v_{\alpha} c_{\alpha}}{\lambda_{\alpha}}\right) \geq \prod_{\alpha \in \mathcal{A}}\left(\frac{v_{\alpha} c_{\alpha}}{\lambda_{\alpha}}\right)^{\lambda_{\alpha}}=\prod_{\alpha \in \mathcal{A}} v_{\alpha}^{\lambda_{\alpha}} \cdot \prod_{\alpha \in \mathcal{A}}\left(\frac{c_{\alpha}}{\lambda_{\alpha}}\right)^{\lambda_{\alpha}} \geq\left|w_{\beta}\right| \prod_{\alpha \in \mathcal{A}}\left(\frac{c_{\alpha}}{\lambda_{\alpha}}\right)^{\lambda_{\alpha}},
$$

which implies

$$
(v, w)(f)=\sum_{\alpha \in \mathcal{A}} v_{\alpha} c_{\alpha}+w_{\beta} d \geq\left|w_{\beta}\right|\left(\prod_{\alpha \in \mathcal{A}}\left(\frac{c_{\alpha}}{\lambda_{\alpha}}\right)^{\lambda_{\alpha}}+\operatorname{sgn}\left(w_{\beta}\right) d\right) .
$$

In the even case, we have $\operatorname{sgn}\left(w_{\beta}\right)=+1$ by $(c)$, and the right expression in (8) is non-negative, because $f$ is a non-negative AG function. In the odd case, observe that then the non-negativity of $f$ yields non-negativity of the right expression in (8) as well.

Remark 3.7. In the beginning of this section, we identified the dual space of $\mathbb{R}[\mathcal{A}, \mathcal{B}]$ with $\mathbb{R}^{(\mathcal{A}, \mathcal{B})}$. Using the reverse identification and associating for every $v \in \mathbb{R}^{(\mathcal{A}, \mathcal{B})}$ a function of form (1), we can identify the dual cones $\left(P_{A, \beta}^{\text {even }}\right)^{*}$ resp. $\left(P_{A, \beta}^{\text {odd }}\right)^{*}$ with the cones of all functions of the form (1) with coefficients in $\left(P_{A, \beta}^{\text {even }}\right)^{*}$ resp. $\left(P_{A, \beta}^{\text {odd }}\right)^{*}$. If $\beta \in \operatorname{conv}(\mathcal{A})$, then by Theorem 2.8 and Lemma 3.6, it is easy to see that $\left(P_{A, \beta}^{\text {even }}\right)^{*} \subseteq P_{A, \beta}^{\text {even }}$ and $\left(P_{A, \beta}^{\text {odd }}\right)^{*} \subseteq P_{A, \beta}^{\text {odd }}$. 
In particular, this means that every function of the form (1) with coefficients in $\left(P_{A, \beta}^{\text {even }}\right)^{*}$ resp. $\left(P_{A, \beta}^{\text {odd }}\right)^{*}$ is non-negative. Hence, $C_{\mathcal{S}}(\mathcal{A}, \mathcal{B})^{*} \subseteq C_{\mathcal{S}}(\mathcal{A}, \mathcal{B})$.

The reverse inclusion does not hold in general. With $\mathcal{A}=\{0,2\}, \beta=1, v_{0}=v_{2}=1$ and $v_{1}=-2$, we obtain $\left(P_{A, \beta}^{\text {even }}\right)^{*} \subsetneq P_{A, \beta}^{\text {even }}$ as well as $\left(P_{A, \beta}^{\text {odd }}\right)^{*} \subsetneq P_{A, \beta}^{\text {odd }}$. Setting $\mathcal{B}=\{1\}$ it follows that $C_{\mathcal{S}}(\mathcal{A}, \mathcal{B})^{*} \subsetneq C_{\mathcal{S}}(\mathcal{A}, \mathcal{B})$

In addition, we need the following lemma for the proof of Theorem 3.5. Here, for $\lambda \in \mathbb{R}_{+}^{\mathcal{A}}$, denote by $\operatorname{supp}(\lambda)=\left\{\alpha \in \mathcal{A} \mid \lambda_{\alpha} \neq 0\right\}$ its support.

Lemma 3.8 (Essentially Lemma 8 of [21]). Let $\mathcal{A} \subseteq \mathbb{R}^{n}$ be a non-empty finite set and $\beta \in$ $\operatorname{conv}(\mathcal{A})$. Then every $\lambda \in \Lambda(\mathcal{A}, \beta)$ can be written as a sum

$$
\lambda=\sum_{j=1}^{k} \mu_{j} \lambda^{(j)}
$$

with $k \geq 1, \mu \in \mathbb{R}_{+}^{k}, \sum_{j=1}^{k} \mu_{j}=1$ and $\lambda^{(j)} \in \Lambda(\mathcal{A}, \beta)$ for all $j$, such that the support of each $\lambda^{(j)}$ is affinely independent.

Proof. Since the polytope $\Lambda(\mathcal{A}, \beta)$ is the convex hull of its vertices, it suffices to show that the support of every vertex of $\Lambda(\mathcal{A}, \beta)$ is an affinely independent set.

Let $\lambda$ be a vertex of $\Lambda(\mathcal{A}, \beta)$ and $\mathcal{A}^{\prime}:=\left\{\alpha \mid \lambda_{\alpha}>0\right\}$ be its support. Assume to the contrary that $\mathcal{A}^{\prime}$ is affinely dependent. Then there exists $\mu \in \mathbb{R}^{\mathcal{A}} \backslash\{0\}$ with $\sum_{\alpha \in \mathcal{A}^{\prime}} \mu_{\alpha}=0, \sum_{\alpha \in \mathcal{A}^{\prime}} \mu_{\alpha} \alpha=0$ and $\mu_{\alpha}=0$ for $\alpha \notin \mathcal{A}^{\prime}$. Since $\lambda_{\alpha}>0$ for all $\alpha \in \mathcal{A}^{\prime}$, for sufficiently small $\epsilon>0$ both $\lambda+\epsilon \mu$ and $\lambda-\epsilon \mu$ are contained in $\Lambda(\mathcal{A}, \beta)$. But this implies that $\lambda=\frac{1}{2}(\lambda+\epsilon \mu)+\frac{1}{2}(\lambda-\epsilon \mu)$ is not a vertex of $\Lambda(\mathcal{A}, \beta)$, a contradiction.

Proof of Theorem 3.5. (1): Since $|\mathbf{x}|^{\alpha} \in C_{\mathcal{S}}(\mathcal{A}, \mathcal{B})$ for every $\alpha \in \mathcal{A}$, every $v \in C_{\mathcal{S}}(\mathcal{A}, \mathcal{B})^{*}$ satisfies

$$
0 \leq(v, w)\left(|\mathbf{x}|^{\alpha}\right)=v_{\alpha}
$$

(2): The implications (b) $\Longrightarrow(c) \Longrightarrow(d)$ are trivial. For the equivalence of (a) and (b) note that

$$
C_{\mathcal{S}}(\mathcal{A}, \mathcal{B})^{*}=\bigcap_{\alpha \in \mathcal{A}}\left(P_{\mathcal{A} \backslash\{\alpha\}, \alpha}^{\mathrm{even}}\right)^{*} \cap \bigcap_{\beta \in \mathcal{B}}\left(P_{\mathcal{A}, \beta}^{\mathrm{odd}}\right)^{*},
$$

because Minkowski sum and intersection are dual operations (see, e.g., [25], Theorem 1.6.3). Hence, the claim follows with Lemma 3.6. It remains to show $(\mathrm{c}) \Longrightarrow(\mathrm{b})$ and $(\mathrm{d}) \Longrightarrow(\mathrm{c})$.

(c) $\Longrightarrow$ (b): Let $\beta \in \mathcal{A}$ and $\lambda \in \Lambda(\mathcal{A}, \beta)$. By Lemma 3.8, we can decompose $\lambda$ as $\lambda=$ $\sum_{j=1}^{k} \mu_{j} \lambda^{(j)}$ with $k \geq 1, \mu \in \mathbb{R}_{+}^{k}, \sum_{j=1}^{k} \mu_{j}=1$ and $\lambda^{(1)}, \ldots, \lambda^{(k)} \in \Lambda(\mathcal{A}, \beta)$, such that the support of each $\lambda^{(j)}$ is affinely independent. Now the claim follows from

$$
\ln \left|v_{\beta}\right|=\sum_{j=1}^{k} \mu_{j} \ln \left|v_{\beta}\right| \stackrel{(c)}{\leq} \sum_{j=1}^{k} \mu_{j} \sum_{\alpha} \lambda_{\alpha}^{(j)} \ln v_{\alpha}=\sum_{\alpha} \ln v_{\alpha} \sum_{j=1}^{k} \mu_{j} \lambda_{\alpha}^{(j)}=\sum_{\alpha} \lambda_{\alpha} \ln v_{\alpha} .
$$

For $\beta \in \mathcal{B}$, the proof is analogous by considering $w_{\beta}$ instead of $v_{\beta}$. 
$(\mathrm{d}) \Longrightarrow(\mathrm{c})$ : We start with the even case and proceed by induction on $r=r_{\mathrm{e}}(A, \beta)$. Since the base case $r=0$ captures exactly the reduced circuits, there is nothing to prove in this case. Now consider an even circuit $(A, \beta) \in I(\mathcal{A}, \mathcal{A})$ with $r_{\mathrm{e}}(A, \beta)>0$. Then there exists a $\beta^{\prime} \in$ $\operatorname{conv}(A) \cap \mathcal{A}$ with $\beta^{\prime} \notin A$ and $\beta^{\prime} \neq \beta$. Set $\lambda:=\lambda(A, \beta)$ and $\lambda^{\prime}:=\lambda\left(A, \beta^{\prime}\right)$.

Let $\tau \geq 0$ be the maximal real number with $\tilde{\lambda}:=\lambda-\tau \lambda^{\prime} \in \mathbb{R}_{+}^{A}$. This number exists clearly, and we have $\tau \leq 1$ because the coordinate sums of $\lambda$ and $\lambda^{\prime}$ are equal. Further, it holds that $\tau>0$ because all components of $\lambda$ are positive.

Similarly, let $\tau^{\prime}$ be the maximal real number with $\tilde{\lambda}^{\prime}:=\lambda^{\prime}-\tau^{\prime} \lambda \in \mathbb{R}_{+}^{A}$. As above, it holds that $0 \leq \tau^{\prime} \leq 1$. Moreover, note that $\beta \neq \beta^{\prime}$ implies $\tau, \tau^{\prime}<1$. The construction gives

$$
\begin{aligned}
\beta & =\sum_{\alpha \in A} \tilde{\lambda}_{\alpha} \alpha+\tau \beta^{\prime}, & \sum_{\alpha \in A} \tilde{\lambda}_{\alpha}+\tau=1, \\
\beta^{\prime}=\sum_{\alpha \in A} \tilde{\lambda}_{\alpha}^{\prime} \alpha+\tau^{\prime} \beta & \text { and } \quad & \sum_{\alpha \in A} \tilde{\lambda}_{\alpha}^{\prime}+\tau^{\prime}=1 .
\end{aligned}
$$

Note that at least one of the entries of $\tilde{\lambda}$ is zero, and moreover, $\tau^{\prime}$ or at least one of the entries of $\widetilde{\lambda}^{\prime}$ is zero. Define two new even circuits $\left(A_{1}, \beta\right)$ and $\left(A_{2}, \beta^{\prime}\right)$ with $A_{1}:=\operatorname{supp}(\tilde{\lambda}) \cup\left\{\beta^{\prime}\right\}$ and

$$
A_{2}:= \begin{cases}\operatorname{supp}\left(\widetilde{\lambda}^{\prime}\right) \cup\{\beta\} & \text { if } \tau^{\prime}>0, \\ \operatorname{supp}\left(\widetilde{\lambda}^{\prime}\right) & \text { if } \tau^{\prime}=0 .\end{cases}
$$

We observe $\operatorname{conv}\left(A_{1}\right) \subsetneq \operatorname{conv}(A)$, and since $\beta^{\prime}$ is not counted towards $r_{\mathrm{e}}\left(A_{1}, \beta\right)$, it follows that $r_{\mathrm{e}}\left(A_{1}, \beta\right)<r_{\mathrm{e}}(A, \beta)$. Similarly, since $\operatorname{conv}\left(A_{2}\right) \subseteq \operatorname{conv}(A)$ and $\beta^{\prime}$ is not counted towards $r_{\mathrm{e}}\left(A_{2}, \beta^{\prime}\right)$, we obtain $r_{\mathrm{e}}\left(A_{2}, \beta^{\prime}\right)<r_{\mathrm{e}}(A, \beta)$. Hence, by induction,

$$
\begin{aligned}
& \ln \left(\left|v_{\beta}\right|\right) \leq \sum_{\alpha \in A} \tilde{\lambda}_{\alpha} \ln \left(v_{\alpha}\right)+\tau \ln \left(v_{\beta^{\prime}}\right) \quad \text { and } \\
& \ln \left(\left|v_{\beta^{\prime}}\right|\right) \leq \sum_{\alpha \in A} \tilde{\lambda}_{\alpha}^{\prime} \ln \left(v_{\alpha}\right)+\tau^{\prime} \ln \left(v_{\beta}\right) .
\end{aligned}
$$

Note that $v_{\beta^{\prime}} \geq 0$ and $v_{\beta} \geq 0$. Adding $\tau$ times (10) to (9) gives, due to $\tilde{\lambda}+\tau \widetilde{\lambda}^{\prime}=\left(1-\tau \tau^{\prime}\right) \lambda$, the uniform inequality

$$
0 \leq\left(1-\tau \tau^{\prime}\right)\left(\sum_{\alpha \in A} \lambda_{\alpha} \ln v_{\alpha}-\ln \left|v_{\beta}\right|\right)
$$

Since $1-\tau \tau^{\prime}>0$, this proves the claim.

For the odd case, we proceed by induction on $r_{\mathrm{o}}(A, \beta)$, and the base case consists again of the reduced circuits. Fix an odd circuit $(A, \beta) \in I(\mathcal{A}, \mathcal{B})$ with $r_{\mathrm{o}}(A, \beta)>0$. Again, there exists a $\beta^{\prime} \in \operatorname{conv}(A) \cap \mathcal{A}$ with $\beta^{\prime} \notin A$, but this time $\beta^{\prime}=\beta$ is possible.

We define $\lambda, \lambda^{\prime}, \tau$ and $\widetilde{\lambda}=\lambda-\tau \lambda^{\prime}$ as above. This time, $\tau=1$ is possible. Further, we set $\tau^{\prime}:=0$ and (thus) $\widetilde{\lambda}^{\prime}:=\lambda^{\prime}$. We define the new circuits $\left(A_{1}, \beta\right)$ and $\left(A_{2}, \beta^{\prime}\right)$ as above, where this time 
$\left(A_{1}, \beta\right)$ is odd and $\left(A_{2}, \beta^{\prime}\right)$ is even. Since $r_{\mathrm{o}}\left(A_{1}, \beta\right)<r_{\mathrm{o}}(A, \beta)$ as above, we obtain

$$
\begin{aligned}
& \ln \left(\left|w_{\beta}\right|\right) \leq \sum_{\alpha \in A} \tilde{\lambda}_{\alpha} \ln \left(v_{\alpha}\right)+\tau \ln \left(v_{\beta^{\prime}}\right) \quad \text { and } \\
& \ln \left(\left|v_{\beta^{\prime}}\right|\right) \leq \sum_{\alpha \in A} \tilde{\lambda}_{\alpha}^{\prime} \ln \left(v_{\alpha}\right),
\end{aligned}
$$

where the second inequality follows since we have already shown $(d) \Longrightarrow(c)$ for even circuits. As above, we add $\tau$ times (12) to (11) to obtain the desired inequality.

A description of the dual of the SONC cone was obtained in [9, Theorem 3.1], and a description of the dual of the SAGE cone in [4, Proposition 2.4]. Both descriptions are based on projections and differ from the one in Theorem 3.5. For completeness, we show here that they are in fact equivalent.

Proposition 3.9. Let $\emptyset \neq \mathcal{A} \subseteq \mathbb{R}^{n}$ be a finite set and $\beta \in \operatorname{conv}(\mathcal{A})$. For $v \in \mathbb{R}_{+}^{\mathcal{A}}$ and $w_{\beta} \in \mathbb{R}$, the following are equivalent:

(1) $\forall \lambda \in \Lambda(\mathcal{A}, \beta): \ln \left|w_{\beta}\right| \leq \sum_{\alpha \in \mathcal{A}} \lambda_{\alpha} \ln \left(v_{\alpha}\right)$.

(2) $\exists \tau \in \mathbb{R}^{n}, \forall \alpha \in \mathcal{A}:\left|w_{\beta}\right| \ln \left(\frac{\left|w_{\beta}\right|}{v_{\alpha}}\right) \leq(\beta-\alpha)^{T} \tau$.

(3) $\exists v^{*} \geq\left|w_{\beta}\right|, \exists \tau \in \mathbb{R}^{n}, \forall \alpha \in \mathcal{A}: v^{*} \ln \left(\frac{v^{*}}{v_{\alpha}}\right) \leq(\beta-\alpha)^{T} \tau$.

In this proposition, statement (1) is the one we used earlier, statement (2) is the description of the dual SAGE cone used in [4], and statement (3) in conjunction with Theorem 3.5(c) is the description of the dual SONC cone used in [9].

Proof. If $w_{\beta}=0$ then all three conditions hold. Moreover, if $v_{\alpha}=0$ for some $\alpha \in \mathcal{A}$, then it is easy to see that all three conditions hold if and only if $w_{\beta}=0$. Thus we may assume that $w_{\beta} \neq 0$ and $v_{\alpha} \neq 0$ for all $\alpha \in \mathcal{A}$. We will show the equivalence via the following variant of statement (2),

(2') $\exists \tau \in \mathbb{R}^{n}, \forall \alpha \in \mathcal{A}: \ln \left(\frac{\left|w_{\beta}\right|}{v_{\alpha}}\right) \leq(\alpha-\beta)^{T} \tau$.

$(1) \Longleftrightarrow\left(2^{\prime}\right)$ : Consider (2') as the feasibility of a linear system of inequalities in $\tau$. (2') is satisfied if and only if its Farkas alternative system (in the version of Proposition 1.7 of [31])

$$
\exists \lambda \in \mathbb{R}_{+}^{\mathcal{A}}: \quad \sum_{\alpha \in \mathcal{A}} \lambda_{\alpha}(-\alpha+\beta)=0 \text { and } \sum_{\alpha \in \mathcal{A}} \lambda_{\alpha} \cdot\left(-\ln \left(\frac{\left|w_{\beta}\right|}{v_{\alpha}}\right)\right)<0
$$

does not have a solution.

We can normalize $\lambda$ so that all its components sum to 1 . Hence, the alternative system simplifies to

$$
\sum_{\alpha \in \mathcal{A}} \lambda_{\alpha} \ln \left|w_{\beta}\right|>\sum_{\alpha \in \mathcal{A}} \lambda_{\alpha} v_{\alpha}>0
$$

i.e., to $\ln \left|w_{\beta}\right|>\sum_{\alpha \in \mathcal{A}} \lambda_{\alpha} v_{\alpha}$. Since this is the opposite of (1), the equivalence of (1) and (2') follows.

$\left(2^{\prime}\right) \Longrightarrow(2)$ : We obtain (2) from (2') by multiplying with $\left|w_{\beta}\right|$ and replacing $\left|w_{\beta}\right| \tau$ by $-\tau$.

$(2) \Longrightarrow(3)$ : This is trivial. 
$(3) \Longrightarrow\left(2^{\prime}\right)$ : We have that $v^{*} \geq\left|w_{\beta}\right|>0$ and thus we may divide the inequality in (3) by $v^{*}$ to obtain

$$
\exists \tau^{\prime} \in \mathbb{R}^{n}, \forall \alpha \in \mathcal{A}: \ln \left(\frac{v^{*}}{v_{\alpha}}\right) \leq(\beta-\alpha)^{T} \tau^{\prime},
$$

where $\tau^{\prime}=\tau / v^{*}$. Note that the left-hand side of the inequality is monotonous in $v^{*}$, and hence,

$$
\ln \left(\frac{\left|w_{\beta}\right|}{v_{\alpha}}\right) \leq \ln \left(\frac{v^{*}}{v_{\alpha}}\right) \leq(\beta-\alpha)^{T} \tau^{\prime} .
$$

We further replace $\tau^{\prime}$ by $-\tau^{\prime}$ to obtain $\left(2^{\prime}\right)$.

\section{Applications of the dual cone}

4.1. Non-negative AG functions are sums of non-negative circuit functions. As a first application of our description of the dual cone, we prove the following generalization of [21, Theorem 4].

Proposition 4.1. Let $\emptyset \neq \mathcal{A} \subseteq \mathbb{R}^{n}$ and $\mathcal{B} \subseteq \mathbb{N}^{n} \backslash(2 \mathbb{N})^{n}$ be finite sets. For every $f \in C_{\mathcal{S}}(\mathcal{A}, \mathcal{B})$, the following statements hold.

(1) $f$ can be written as a sum of non-negative circuit functions whose supports are contained in $\operatorname{supp} f$.

(2) $f$ can be written as a sum of non-negative circuit functions supported on reduced circuits in $C_{\mathcal{S}}(\mathcal{A}, \mathcal{B})$.

Note that in statement (2), the support of the reduced circuits does not need to be contained in the support of $f$. The following example shows a situation, in which this phenomenon happens.

Example 4.2. Let $\mathcal{A}:=\{0,2,4\}$ and $\mathcal{B}:=\{1\}$. Consider the non-negative circuit function $f=|x|^{0}-4 \cdot 3^{-3 / 4} x+|x|^{4}=1-4 \cdot 3^{-3 / 4} x+x^{4}$. Its support $(\{0,4\}, 1)$ is not reduced with respect to $\mathcal{A}, \mathcal{B}$, and indeed, we can write $f$ as sum

$$
\begin{aligned}
f & =\left(\frac{2}{3}-4 \cdot 3^{-3 / 4} x+\frac{2}{3} \sqrt{3} x^{2}\right)+\left(\frac{1}{3}-\frac{2}{3} \sqrt{3} x^{2}+x^{4}\right) \\
& =\left(\frac{2}{3}|x|^{0}-4 \cdot 3^{-3 / 4} x+\frac{2}{3} \sqrt{3}|x|^{2}\right)+\left(\frac{1}{3}|x|^{0}-\frac{2}{3} \sqrt{3}|x|^{2}+|x|^{4}\right)
\end{aligned}
$$

of non-negative circuit functions, whose supports $(\{0,2\}, 1)$ and $(\{0,4\}, 2)$ are reduced. Note that the coefficient of $|x|^{2}$ cancels in the sum.

Proof of Proposition 4.1. By Lemma 3.6 and part (c) of Theorem 3.5, the dual of the $\mathcal{S}$-cone is

$$
C_{\mathcal{S}}(\mathcal{A}, \mathcal{B})^{*}=\bigcap_{(A, \beta) \in I(\mathcal{A}, \mathcal{A})}\left(P_{A \backslash\{\beta\}, \beta}^{\text {even }}\right)^{*} \cap \bigcap_{(A, \beta) \in I(\mathcal{A}, \mathcal{B})}\left(P_{A, \beta}^{\text {odd }}\right)^{*} .
$$

Let $f \in C_{\mathcal{S}}(\mathcal{A}, \mathcal{B})$ and assume that the support of $f$ is given by $\mathcal{A}^{\prime} \subseteq \mathcal{A}$ and $\mathcal{B}^{\prime} \subseteq B$. By Proposition 2.7, $f \in C_{\mathcal{S}}\left(\mathcal{A}^{\prime}, \mathcal{B}^{\prime}\right)$. Apply (13) on the sub-cone $C_{\mathcal{S}}\left(\mathcal{A}^{\prime}, \mathcal{B}^{\prime}\right)$ and dualize that 
identity. Using that $C_{\mathcal{S}}\left(\mathcal{A}^{\prime}, \mathcal{B}^{\prime}\right)^{* *}=C_{\mathcal{S}}\left(\mathcal{A}^{\prime}, \mathcal{B}^{\prime}\right)$ (because the cone is closed, Proposition 2.10) then yields

$$
f \in \sum_{(A, \beta) \in I(\mathcal{A}, \mathcal{A})} P_{A \backslash\{\beta\}, \beta}^{\mathrm{even}}+\sum_{(A, \beta) \in I(\mathcal{A}, \mathcal{B})} P_{A, \beta}^{\text {odd }} .
$$

This shows part (1).

Part (2) then follows from part (d) of Theorem 3.5. Note that in this case we cannot restrict the sets of exponents to $\mathcal{A}^{\prime}$ and $\mathcal{B}^{\prime}$ as it depends on the choice of $\mathcal{A}$ and $\mathcal{B}$ whether a circuit is reduced or not.

Remark 4.3. If we demand $\operatorname{supp}(f)=\mathcal{A} \cup \mathcal{B}$, we obtain the same statement about the support in (2) as in (1).

4.2. Extreme rays of the $\mathcal{S}$-cone. Our next application of our description of the dual cone is a precise characterization of the extreme rays of $C_{\mathcal{S}}(\mathcal{A}, \mathcal{B})$. Even for the specific case of the SAGE cone, this sharpens the result in [21, Theorem 4], where the necessary condition is that every extreme ray of the SAGE cone is supported on a single coordinate or on a circuit. The essential concept for this characterization is provided by the reduced circuits.

Let $\emptyset \neq \mathcal{A} \subseteq \mathbb{R}^{n}$ and $\mathcal{B} \subseteq \mathbb{N}^{n} \backslash(2 \mathbb{N})^{n}$ be finite sets and write shortly $\lambda=\lambda(A, \beta)$. For $(A, \beta) \in I(\mathcal{A}, \mathcal{A})$ let

$$
E_{\mathrm{e}}(A, \beta):=\left\{\sum_{\alpha \in A} c_{\alpha}|\mathbf{x}|^{\alpha}-\prod_{\alpha \in A}\left(\frac{c_{\alpha}}{\lambda_{\alpha}}\right)^{\lambda_{\alpha}}|\mathbf{x}|^{\beta} \mid c \in \mathbb{R}_{>0}^{A}\right\},
$$

for $(A, \beta) \in I(\mathcal{A}, \mathcal{B})$ let

$$
E_{\mathrm{o}}(A, \beta):=\left\{\sum_{\alpha \in A} c_{\alpha}|\mathbf{x}|^{\alpha} \pm \prod_{\alpha \in A}\left(\frac{c_{\alpha}}{\lambda_{\alpha}}\right)^{\lambda_{\alpha}} \mathbf{x}^{\beta} \mid c \in \mathbb{R}_{>0}^{A}\right\},
$$

and for $\beta \in \mathcal{A}$ let

$$
E_{1}(\beta):= \begin{cases}\mathbb{R}_{+} \cdot|\mathbf{x}|^{\beta} & \text { if } \beta \in \mathcal{A} \backslash \mathcal{B}, \\ \mathbb{R}_{+} \cdot\left(|\mathbf{x}|^{\beta} \pm \mathbf{x}^{\beta}\right) & \text { if } \beta \in \mathcal{A} \cap \mathcal{B} .\end{cases}
$$

$E_{\mathrm{e}}(A, \beta)$ and $E_{\mathrm{o}}(A, \beta)$ are the (even and odd) non-negative circuit functions, for which the inequality $(7)$ on the circuit number holds with equality. $E_{1}(\beta)$ provides the special case for circuits supported on a single element.

Proposition 4.4. For finite sets $\emptyset \neq \mathcal{A} \subseteq \mathbb{R}^{n}$ and $\mathcal{B} \subseteq \mathbb{N}^{n} \backslash(2 \mathbb{N})^{n}$, the set $\mathcal{E}(\mathcal{A}, \mathcal{B})$ of extreme rays of $C_{\mathcal{S}}(\mathcal{A}, \mathcal{B})$ is

$$
\mathcal{E}(\mathcal{A}, \mathcal{B})=\left(\bigcup_{\substack{(A, \beta) \in I(\mathcal{A}, \mathcal{A}), r_{\mathrm{e}}(A, \beta)=0,|A|>1}} E_{\mathrm{e}}(A, \beta)\right) \cup\left(\bigcup_{\begin{array}{c}
(A, \beta) \in I(\mathcal{A}, \mathcal{B}), \\
r_{\mathrm{o}}(A, \beta)=0,|A|>1
\end{array}} E_{\mathrm{o}}(A, \beta)\right) \cup\left(\bigcup_{\beta \in \mathcal{A}} E_{1}(\beta)\right) .
$$

Here, recall from Definition 3.4 that an even (respectively odd) circuit is reduced if and only if $r_{\mathrm{e}}(A, \beta)=0$ (respectively $r_{\mathrm{o}}(A, \beta)=0$ ). The following example shows that the case distinctions are indeed necessary. 
Example 4.5. For $\mathcal{A}:=\{0,1,2\}$ and $\mathcal{B}:=\{1\}$, the sets of (even resp. odd) circuits are

$$
\begin{aligned}
I(\mathcal{A}, \mathcal{A}) & =\{(\{0,2\}, 1),(\{0\}, 0),(\{1\}, 1),(\{2\}, 2)\} \text { and } \\
I(\mathcal{A}, \mathcal{B}) & =\{(\{1\}, 1),(\{0,2\}, 1)\} .
\end{aligned}
$$

We have a closer look at those elements which are both even and odd circuits.

(1) The circuit $(\{0,2\}, 1)$ is reduced as an even circuit and non-reduced as an odd circuit. In the context of extreme rays this is necessary. The even circuit function $a^{2}-2 a b|x|+b^{2} x^{2}$ is an element of an extreme ray, but for the odd circuit function $a^{2} \pm 2 a b x+b^{2} x^{2}$ we have

$$
a^{2} \pm 2 a b x+b^{2} x^{2}=\left(a^{2}-2 a b|x|+b^{2} x^{2}\right)+2 a b(|x| \pm x)
$$

and hence this is not an extreme ray of $C_{\mathcal{S}}(\mathcal{A}, \mathcal{B})$.

(2) Further, it holds that

$$
|x|=\frac{1}{2}(|x|+x)+\frac{1}{2}(|x|-x),
$$

so $(\{1\}, 1)$ does not support an even extreme ray but in fact it does support an odd extreme ray.

Again, we obtain corollaries for the special cases of SONC polynomials and of the SAGE cone.

Corollary 4.6. Let $\emptyset \neq \mathcal{A} \subseteq \mathbb{N}^{n}$ be a finite set and write shortly $\lambda=\lambda(A, \beta)$. The set $\mathcal{E}(\mathcal{A})$ of extreme rays of the cone of SONC-polynomials with support in $\mathcal{A}$ is

$$
\begin{aligned}
& \mathcal{E}(\mathcal{A})=\bigcup_{\substack{(A, \beta) \in I\left(\mathcal{A} \cap(2 \mathbb{N})^{n}, \mathcal{A}\right), r_{\mathrm{e}}(A, \beta)=0,|A|>1}}\left\{\sum_{\alpha \in A} c_{\alpha} x^{\alpha}-\prod_{\alpha \in A}\left(\frac{c_{\alpha}}{\lambda_{\alpha}}\right)^{\lambda_{\alpha}} x^{\beta} \mid c \in \mathbb{R}_{>0}^{A}\right\} \\
& \cup \underset{\substack{(A, \beta) \in I\left(\mathcal{A} \cap(2 \mathbb{N})^{n}, \mathcal{A}\right), r_{\mathrm{o}}(A, \beta)=0,|A|>1, \beta \in \mathcal{A} \backslash(2 \mathbb{N})^{n}}}{U}\left\{\sum_{\alpha \in A} c_{\alpha} x^{\alpha}+\prod_{\alpha \in A}\left(\frac{c_{\alpha}}{\lambda_{\alpha}}\right)^{\lambda_{\alpha}} x^{\beta} \mid c \in \mathbb{R}_{>0}^{A}\right\} \\
& \cup \bigcup_{\beta \in \mathcal{A} \cap(2 \mathbb{N})^{n}} \mathbb{R}_{+} \cdot x^{\beta} .
\end{aligned}
$$

Corollary 4.7. Let $\emptyset \neq \mathcal{A} \subseteq \mathbb{R}^{n}$ be a finite set, $\mathcal{B}=\emptyset$ and write $\lambda=\lambda(A, \beta)$. The set $\mathcal{E}(\mathcal{A}, \mathcal{B})$ of extreme rays of the cone of $S A G E$ functions with support in $\mathcal{A}$ is

$$
\begin{aligned}
\mathcal{E}(\mathcal{A}, \mathcal{B})= & \bigcup_{\substack{(A, \beta) \in I(\mathcal{A}, \mathcal{A}), r_{\mathrm{e}}(A, \beta)=0,|\mathcal{A}|>1}} E_{\mathrm{e}}(A, \beta) \cup \bigcup_{\beta \in \mathcal{A}} E_{1}(\beta) \\
= & \bigcup_{\substack{(A, \beta) \in I(\mathcal{A}, \mathcal{A}), r_{\mathrm{e}}(A, \beta)=0,|A|>1}}\left\{\sum_{\alpha \in A} c_{\alpha} \exp \left(y^{T} \alpha\right)-\prod_{\alpha \in A}\left(\frac{c_{\alpha}}{\lambda_{\alpha}}\right)^{\lambda_{\alpha}} \exp \left(y^{T} \beta\right) \mid c \in \mathbb{R}_{>0}^{\mathcal{A}}\right\} \\
& \cup \bigcup_{\beta \in \mathcal{A}}\left\{c \exp \left(y^{T} \beta\right) \mid c \in \mathbb{R}_{+}\right\} .
\end{aligned}
$$


Example 4.8. As an example corresponding to the SAGE setting, let $\mathcal{A}=\{0,1,2,4\}, \mathcal{B}=\emptyset$ and $f:=|x|^{0}-4 \cdot 3^{-3 / 4} x^{1}+|x|^{4}$ be a non-negative circuit function. With the substitution $x \mapsto \exp (y)$ we obtain the arithmetic-geometric exponential $\tilde{f}=1-4 \cdot 3^{-3 / 4} \exp (y)+\exp (4 y)$, and its support is again not reduced. We write $f$ as a sum

$$
f=\left(1-2 \cdot 3^{1 / 4}|x|+\sqrt{3} x^{2}\right)+\left(\frac{2}{3} 3^{1 / 4}|x|-\sqrt{3} x^{2}+x^{4}\right)
$$

of circuit functions, whose supports $\{0,1,2\}$ and $\{1,2,4\}$ are reduced.

This is different from Example 4.2 in that the exponent 1 is contained in $\mathcal{A}$ rather than $\mathcal{B}$ and thus is treated like an even number in the SAGE setting.

In [21], after Theorem 4, the authors remark that every circuit "supports a family of extreme rays in the SAGE cone." This is not quite correct, as shown by the current example.

For the proof of Proposition 4.4, we will use a variant of Hölder's inequality.

Theorem 4.9 (Theorem 11, p. 22, [11]). Let $n, m \in \mathbb{N}$. Let $\left(a_{i j}\right) \in \mathbb{R}^{n \times m}$ be a matrix and let $\lambda_{1}, \ldots, \lambda_{n} \in \mathbb{R}_{>0}$ with $\sum_{i=1}^{n} \lambda_{i}=1$. Then

$$
\sum_{j=1}^{m} \prod_{i=1}^{n} a_{i j}^{\lambda_{i}} \leq \prod_{i=1}^{n}\left(\sum_{j=1}^{m} a_{i j}\right)^{\lambda_{i}},
$$

and equality holds if and only if either (1) for some $i, a_{i 1}=\cdots=a_{i m}=0$, or (2) the matrix $\left(a_{i j}\right)$ has rank one.

Note that in case (1) both sides of the inequality are zero.

Proof of Proposition 4.4. By Proposition 4.1, every non-negative function $f \in C_{\mathcal{S}}(\mathcal{A}, \mathcal{B})$ can be written as a sum of non-negative circuit functions supported on reduced circuits. Hence, it suffices to show the following two statements:

(a) Every non-negative circuit function supported on a circuit can be written as a sum of nonnegative circuit functions with the same support whose circuit condition is satisfied with equality.

(b) Every function in $\mathcal{E}(\mathcal{A}, \mathcal{B})$ is indeed an extreme ray, i.e., it cannot be written as a sum of other non-negative $\mathrm{AG}$ functions.

(a) Let $f$ be a non-negative circuit function supported on the circuit $(A, \beta)$, whose coefficients are denoted by $\left(c_{\alpha}\right)_{\alpha \in A}$ and $c_{\beta}$.

If $f$ is supported on an odd circuit $(A, \beta) \in I(\mathcal{A}, \mathcal{B})$, then the circuit number $\Theta_{f}$ from (7) satisfies $c_{\beta} \in\left[-\Theta_{f}, \Theta_{f}\right]$. Hence, $f$ is a convex combination of $f_{1}$ and $f_{2}$, where $f_{1}, f_{2}$ have the same support and coefficients as $f$, except for $c_{\beta}^{(1)}=\Theta_{f_{1}}=\Theta_{f}$ and $c_{\beta}^{(2)}=-\Theta_{f_{2}}=-\Theta_{f}$.

If $f$ is supported on an even circuit $(A, \beta) \in I(\mathcal{A}, \mathcal{A})$, then $f$ can be written as the sum of a non-negative circuit function with the same support whose inner coefficient equals the negative of the circuit number and of some function $d|\mathbf{x}|^{\beta}$ for $d>0$. If $\beta \notin \mathcal{B}$, then the latter is contained in $E_{1}(\beta)$. Otherwise, if $\beta \in \mathcal{B}$, then $d|\mathbf{x}|^{\beta}=\frac{d}{2}\left(|\mathbf{x}|^{\beta}+\mathbf{x}^{\beta}\right)+\frac{d}{2}\left(|\mathbf{x}|^{\beta}-\mathbf{x}^{\beta}\right)$, whose two summands are elements of $E_{1}(\beta)$. 
(b) Let $f \in \mathcal{E}(\mathcal{A}, \mathcal{B})$ with coefficients $\left(c_{\alpha}\right)_{\alpha \in \mathcal{A}}$ and $\left(d_{\beta}\right)_{\beta \in \mathcal{B}}$. Assume that $f$ can be decomposed into $f=\sum_{i=1}^{k} f_{i}$ with non-negative AG functions $f_{1}, \ldots, f_{k} \in \mathbb{R}[\mathcal{A}, \mathcal{B}]$. Denote the coefficients of $f_{i}$ by $\left(c_{\alpha}^{(i)}\right)_{\alpha \in \mathcal{A}}$ and $\left(d_{\beta}^{(i)}\right)_{\beta \in \mathcal{B} \text {. }}$

For the duration of this proof, we use the notation $\operatorname{supp}_{\mathrm{e}}(f):=\left\{\alpha \in \mathcal{A} \mid c_{\alpha} \neq 0\right\}$. Moreover, set $\tilde{A}:=\bigcup_{i} \operatorname{supp}_{\mathrm{e}}\left(f_{i}\right)=\left\{\alpha \in \mathcal{A} \mid \exists i: c_{\alpha}^{(i)} \neq 0\right\}$. We claim that $\tilde{A} \subseteq \operatorname{conv}_{\operatorname{supp}_{\mathrm{e}}}(f)$.

To show this, we consider a vertex $\tilde{\alpha}$ of conv $\tilde{A}$. Since $\tilde{\alpha}$ must be an outer exponent of each $f_{i}$ with $c_{\tilde{\alpha}}^{(i)} \neq 0$, we have $c_{\tilde{\alpha}}^{(i)} \geq 0$ for all $i$. It follows that $\sum_{i=1}^{k} c_{\tilde{\alpha}}^{(i)}>0$ and thus $\tilde{\alpha} \in \operatorname{supp}_{\mathrm{e}}(f)$. As this holds for every vertex of $\operatorname{conv} \tilde{A}$, we obtain that $\tilde{A} \subseteq \operatorname{conv} \operatorname{supp}_{\mathrm{e}}(f)$.

Next, we distinguish three cases depending on whether $f \in E_{1}(\beta), f \in E_{\mathrm{e}}(A, \beta)$ or $f \in$ $E_{\mathrm{o}}(A, \beta)$.

Case $f \in E_{1}(\beta), \beta \in \mathcal{A}$ : In this case, $\operatorname{supp}_{\mathrm{e}}\left(f_{i}\right)=\{\beta\}$ for each $i$. Thus, if $\beta \notin \mathcal{B}$ then each $f_{i}$ is a multiple of $|\mathbf{x}|^{\beta}$ and thus a multiple of $f$.

On the other hand, if $\beta \in \mathcal{A} \cap \mathcal{B}$, then w.l.o.g. we can assume that $f=c\left(|\mathbf{x}|^{\beta}+\mathbf{x}^{\beta}\right)$ for some $c>0$. Moreover, each $f_{i}$ is of the form $f_{i}=c_{i}|\mathbf{x}|^{\beta}+d_{i} \mathbf{x}^{\beta}$ with $\left|d_{i}\right| \leq c_{i}$. Then

$$
\sum_{i} d_{i}=c=\sum_{i} c_{i} \geq \sum_{i}\left|d_{i}\right| \geq\left|\sum_{i} d_{i}\right|
$$

and thus $d_{i}=c_{i}$ for each $i$. Hence, all $f_{i}$ are multiples of $f$.

Case $f \in E_{\mathrm{e}}(A, \beta)$ for $(A, \beta) \in I(\mathcal{A}, \mathcal{A})$ with $r_{\mathrm{e}}(A, \beta)=0$ and $|A|>1$ : In this case, our initial considerations imply that $\bigcup_{i} \operatorname{supp}_{\mathrm{e}}\left(f_{i}\right) \subseteq \operatorname{conv}(A)$. Since $(A, \beta)$ is reduced we can also conclude that $\bigcup_{i} \operatorname{supp}_{\mathrm{e}}\left(f_{i}\right) \subseteq A \cup\{\beta\}$. Hence, each $f_{i}$ is of the form

$$
f_{i}=\sum_{\alpha \in A} c_{\alpha}^{(i)}|\mathbf{x}|^{\alpha}+c_{\beta}^{(i)}|\mathbf{x}|^{\beta}+\sum_{\beta^{\prime} \in \mathcal{B}} d_{\beta^{\prime}}^{(i)} \mathbf{x}^{\beta} .
$$

It follows that $c_{\alpha}^{(i)} \geq 0$ for all $i$ and $\alpha \in A$, because otherwise the $f_{i}$ cannot be non-negative.

Next, we claim that

$$
-c_{\beta}^{(i)} \leq \prod_{\alpha \in A}\left(\frac{c_{\alpha}^{(i)}}{\lambda_{\alpha}}\right)^{\lambda_{\alpha}}
$$

for all $i$, where again we write $\lambda=\lambda(A, \beta)$. To prove the claim, we distinguish two cases:

(i) If $c_{\beta}^{(i)} \geq 0$, then it trivially holds that $-c_{\beta}^{(i)} \leq 0 \leq \prod_{\alpha \in A}\left(c_{\alpha}^{(i)} / \lambda_{\alpha}\right)^{\lambda_{\alpha}}$.

(ii) Consider the case that $c_{\beta}^{(i)}<0$. Since $f_{i}$ is a non-negative AG function, it holds that the last sum in (14) vanishes and the claim follows from Theorem 2.8(3).

In the next step, we derive

$$
-c_{\beta}=-\sum_{i=1}^{k} c_{\beta}^{(i)} \stackrel{(\mathrm{a})}{\leq} \sum_{i=1}^{k} \prod_{\alpha \in A}\left(\frac{c_{\alpha}^{(i)}}{\lambda_{\alpha}}\right)^{\lambda_{\alpha}} \stackrel{(\mathrm{b})}{\leq} \prod_{\alpha \in A}\left(\sum_{i=1}^{k} \frac{c_{\alpha}^{(i)}}{\lambda_{\alpha}}\right)^{\lambda_{\alpha}} \stackrel{(\mathrm{c})}{=} \prod_{\alpha \in A}\left(\frac{c_{\alpha}}{\lambda_{\alpha}}\right)^{\lambda_{\alpha}}=-c_{\beta},
$$

where in (a) we use (15), (b) follows from Hölder's Inequality 4.9 and (c) uses that $\sum_{i=1}^{k} c_{\alpha}^{(i)}=c_{\alpha}$. Moreover, by Theorem 4.9 equality in (b) implies that either (1) there exists an $\alpha \in A$ such that $c_{\alpha}^{(i)}$ vanishes for all $i$, or (2) the $|A| \times k$ matrix with entries $c_{\alpha}^{(i)} / \lambda_{\alpha}$ has rank one. However, (1) would imply that $c_{\beta}=0$ which is impossible, thus we are in case (2). Hence, there exist 
scalars $\epsilon_{1}, \ldots, \epsilon_{k} \geq 0$ such that $c_{\alpha}^{(i)}=\epsilon_{i} c_{\alpha}$ for all $i$ and all $\alpha \in A$. Further, equality in (a) implies that

$$
-c_{\beta}^{(i)}=\prod_{\alpha \in A}\left(\frac{c_{\alpha}^{(i)}}{\lambda_{\alpha}}\right)^{\lambda_{\alpha}}=\prod_{\alpha \in A}\left(\epsilon_{i} \frac{c_{\alpha}}{\lambda_{\alpha}}\right)^{\lambda_{\alpha}}=-\epsilon_{i} c_{\beta} .
$$

By (14), it follows that every $f_{i}$ is of the form

$$
f_{i}=\epsilon_{i} f+\text { terms in } \mathcal{B} .
$$

Now, if $\epsilon_{i}=0$ for some $i$, then $f_{i}$ has only terms with exponents in $\mathcal{B}$ and thus it is the zero function or it cannot be non-negative. It follows that $\epsilon_{i}>0$ for all $i$. But this implies that $c_{\beta}^{(i)}=\epsilon_{i} c_{\beta}<0$. Hence, since the $f_{i}$ are AG functions, they cannot have any other terms in $\mathcal{B}$, and thus they are all multiples of $f$.

Case $f \in E_{\mathrm{o}}(A, \beta)$ for $(A, \beta) \in I(\mathcal{A}, \mathcal{B})$ with $r_{\mathrm{o}}(A, \beta)=0$ and $|A|>1$ : In this case, the argument is similar, except that (16) becomes

$$
\left|d_{\beta}\right|=\left|\sum_{i=1}^{k} d_{\beta}^{(i)}\right| \stackrel{(\mathrm{e})}{\leq} \sum_{i=1}^{k}\left|d_{\beta}^{(i)}\right| \leq \sum_{i=1}^{k} \prod_{\alpha \in A}\left(\frac{c_{\alpha}^{(i)}}{\lambda_{\alpha}}\right)^{\lambda_{\alpha}} \leq \prod_{\alpha \in A}\left(\sum_{i=1}^{k} \frac{c_{\alpha}^{(i)}}{\lambda_{\alpha}}\right)^{\lambda_{\alpha}}=\prod_{\alpha \in A}\left(\frac{c_{\alpha}}{\lambda_{\alpha}}\right)^{\lambda_{\alpha}}=\left|d_{\beta}\right| .
$$

Since we have equality in (e), it follows that all terms on the left-hand side of that triangle inequality have the same sign. Since $d_{\beta}=\sum_{i=1}^{k} d_{\beta}^{(i)}$, this implies that each $d_{\beta}^{(i)}$ has the same sign as $d_{\beta}$. Now we also obtain (17). Note that for the vanishing of the terms with exponents in $\mathcal{B} \backslash\{\beta\}$, we can argue as above, or alternatively obtain this directly from the oddness of the $f_{i}$. Altogether, this yields again that all the $f_{i}$ are multiples of $f$.

4.3. Univariate SONC polynomials do not satisfy Putinar's Positivstellensatz. In [8, Section 5], a multivariate example was given to show that the analogue of Putinar's Positivstellensatz does not hold for SONC polynomials. Using Theorem 3.5, we provide a simpler example of this phenomenon, which in addition shows that the analogue of Putinar's result does not even hold for univariate SONC polynomials.

Recall Putinar's Theorem from the theory of sums of squares polynomials ([23], see also, e.g., [17, Theorem 2.14]).

Theorem 4.10. Let $f, g_{1}, \ldots, g_{m} \in \mathbb{R}[\mathbf{x}]$ and assume that the quadratic module

$$
Q\left(g_{1}, \ldots, g_{m}\right):=\left\{p_{0}+\sum_{j=1}^{m} p_{j} g_{j} \text { with sums of squares polynomials } p_{0}, \ldots, p_{m}\right\}
$$

is Archimedean. If $f \in \mathbb{R}[\mathbf{x}]$ is strictly positive on the set $K=\left\{\mathbf{x} \in \mathbb{R}^{n}: g_{j}(\mathbf{x}) \geq 0,1 \leq j \leq\right.$ $m\}$, then $f$ can be written in the form $f=p_{0}+\sum_{j=1}^{m} p_{j} g_{j}$ with sum of squares polynomials $p_{0}, \ldots, p_{m}$.

Here, the Archimedean condition can be defined by the existence of some $N \geq 1$ with $N-$ $\sum_{i=1}^{n} x_{i}^{2} \in Q\left(g_{1}, \ldots, g_{m}\right)$, and it is well known that $Q\left(g_{1}, \ldots, g_{m}\right)$ is Archimedean if $g_{1}, \ldots, g_{m}$ are affine (see, e.g., [17]). 
Theorem 4.11. The univariate polynomial

$$
f:=\left(x-\frac{1}{2}\right)^{4}+\frac{1}{1000}
$$

satisfies $f(x)>0$ for $x \in[0,1]$, but it cannot be written in the form

$$
p_{0}+x p_{1}+(1-x) p_{2}+x(1-x) p_{3}
$$

with $S O N C$ polynomials $p_{0}, p_{1}, p_{2}$ and $p_{3}$.

Note that a SONC analogue of Putinar's Positivstellensatz would even assert a representation of $f$ using only $p_{0}, p_{1}$ and $p_{2}$.

Proof. As a notation, for $r \in \mathbb{N}$ we set $C_{\mathcal{S}}(r):=C_{\mathcal{S}}(\{0,1, \ldots, r\} \cap 2 \mathbb{N},\{0,1, \ldots, r\} \backslash 2 \mathbb{N})$. This is the cone of SONC polynomials of degree up to $r$.

The polynomial $f$ is clearly positive on $[0,1]$ (in fact, on $\mathbb{R}$ ), so we only need to show that it does not have a representation as in the claim.

Assume to the contrary that there exist SONC polynomials $p_{0}, p_{1}, p_{2}, p_{3}$ such that (19) holds. Let $d$ be the maximum of the degrees of the $p_{i}$. Then $f$ is contained in $C_{\mathcal{S}}(d+2)+x C_{\mathcal{S}}(d+$ $1)+(1-x) C_{\mathcal{S}}(d+1)+x(1-x) C_{\mathcal{S}}(d)$. On the other hand, consider the vector

$$
v:=\left(\frac{25}{18}, \frac{5}{9}, \frac{1}{2^{2}}, \frac{1}{2^{3}}, \ldots, \frac{1}{2^{d+2}}\right) \in \mathbb{R}^{\{0, \ldots, d+2\}} .
$$

A direct computation shows that

$$
v(f)=-\frac{1}{288}+\frac{25}{18} \frac{1}{1000}=-\frac{1}{480}<0 .
$$

Hence, once we show that $v$ lies in

$$
\begin{aligned}
&\left(C_{\mathcal{S}}(d+2)+x C_{\mathcal{S}}(d+1)+(1-x) C_{\mathcal{S}}(d+1)+x(1-x) C_{\mathcal{S}}(d)\right)^{*} \\
&=C_{\mathcal{S}}(d+2)^{*} \cap\left(x C_{\mathcal{S}}(d+1)\right)^{*} \cap\left((1-x) C_{\mathcal{S}}(d+1)\right)^{*} \cap\left(x(1-x) C_{\mathcal{S}}(d)\right)^{*}
\end{aligned}
$$

we obtain a contradiction.

For this, note that we only need to consider inequalities involving the first two components of $v$, because apart from those $v$ equals the vector $\left(\left(\frac{1}{2}\right)^{\alpha}\right)_{0 \leq \alpha \leq d+2}$, which is clearly contained in the cone. Further, note that $v \in\left(x C_{\mathcal{S}}(d+1)\right)^{*}$ if and only if the shifted vector $\left(v_{1}, v_{2}, \ldots, v_{d+2}\right) \in \mathbb{R}^{\{0, \ldots, d+1\}}$ where we omitted the 0 -th coordinate lies in $C_{\mathcal{S}}(d+1)^{*}$. Similarly, $v$ lies in $\left((1-x) C_{\mathcal{S}}(d+1)\right)^{*}$ if and only if $\left(v_{0}-v_{1}, v_{1}-v_{2}, \ldots, v_{d+1}-v_{d+2}\right)$ lies in $C_{\mathcal{S}}(d+1)^{*}$, and an analogous description holds for $\left(x(1-x) C_{\mathcal{S}}(d)\right)^{*}$. Using these observations, it is a straightforward computation to verify that $v$ lies in the cone.

4.4. Functions with simplex Newton polytopes. We provide a subclass of functions for which non-negativity coincides with containment in the $\mathcal{S}$-cone $C_{\mathcal{S}}(\mathcal{A}, \mathcal{B})$.

Proposition 4.12. Let $\emptyset \neq \mathcal{A} \subseteq \mathbb{R}^{n}, \mathcal{B} \subseteq \mathbb{N}^{n} \backslash(2 \mathbb{N})^{n}$ be finite sets and $f=\sum_{\alpha \in \mathcal{A}} c_{\alpha}|\mathbf{x}|^{\alpha}+$ $\sum_{\beta \in \mathcal{B}} d_{\beta} \mathbf{x}^{\beta} \in \mathbb{R}[\mathcal{A}, \mathcal{B}]$. Assume that

(1) $\operatorname{conv}(\mathcal{A})$ is a simplex and $\mathcal{B} \subseteq \operatorname{conv}(\mathcal{A})$, 
(2) $c_{\alpha} \leq 0$ for every $\alpha \in \mathcal{A}$ which is not a vertex of $\operatorname{conv}(\mathcal{A})$, and

(3) $d_{\beta} \leq 0$ for every $\beta \in \mathcal{B}$.

Then $f$ is non-negative if and only if $f \in C_{\mathcal{S}}(\mathcal{A}, \mathcal{B})$. In this case, $f$ can be written as a sum of circuit functions using only vertices of $\operatorname{conv}(\mathcal{A})$ as outer exponents.

This has been shown for SONC polynomials under a slightly stronger hypothesis in [13, Theorem 5.5]. Moreover, the analogous statement in the SAGE setting has been obtained in [21, Theorem 10]. We provide a simple proof using Theorem 3.5 as well as the following lemma.

Lemma 4.13. Let $\emptyset \neq A \subseteq \mathbb{R}^{n}$ be affinely independent and let $v \in \mathbb{R}_{>0}^{A}$. Then there exists a point $\mathbf{p} \in \mathbb{R}_{>0}^{n}$ and a scalar $\tau \in \mathbb{R}_{>0}$ such that $v_{\alpha}=\tau \mathbf{p}^{\alpha}$ for all $\alpha \in A$.

Proof. Since $A$ is affinely independent, there exists an affine map $\ell: \mathbb{R}^{n} \rightarrow \mathbb{R}$ such that $\ell(\alpha)=$ $\ln \left(v_{\alpha}\right)$ for all $\alpha \in A$. Explicitly, there exists a $\mathbf{w} \in \mathbb{R}^{n}$ and $c \in \mathbb{R}$ with $\mathbf{w}^{T} \alpha+c=\ln \left(v_{\alpha}\right)$ for all $\alpha \in A$. We set $\tau:=\exp (c)$ and $p_{i}:=\exp \left(w_{i}\right)$ for $1 \leq i \leq n$. A straightforward computation shows that $\mathbf{p}:=\left(p_{1}, \ldots, p_{n}\right)$ and $\tau$ satisfy our claim:

$$
v_{\alpha}=\exp (\ell(\alpha))=\exp \left(\sum_{i} \alpha_{i} w_{i}+c\right)=\exp (c) \prod_{i} \exp \left(w_{i}\right)^{\alpha_{i}}=\tau \prod_{i} p_{i}^{\alpha_{i}}=\tau \mathbf{p}^{\alpha} .
$$

Proof of Proposition 4.12. For the nontrivial direction, let $f$ be non-negative and denote by $V \subseteq \mathcal{A}$ the set of vertices of $\operatorname{conv}(\mathcal{A})$. We show that $f$ is contained in the sub-cone

$$
C:=\sum_{\alpha \in \mathcal{A} \backslash V} P_{V, \alpha}^{\text {even }}+\sum_{\beta \in \mathcal{B}} P_{V, \beta}^{\text {odd }} \subseteq C_{\mathcal{S}}(\mathcal{A}, \mathcal{B}) .
$$

Let $(v, w)$ be an arbitrary element of $C^{*}=\bigcap_{\alpha \in \mathcal{A} \backslash V}\left(P_{V, \alpha}^{\text {even }}\right)^{*} \cap \bigcap_{\beta \in \mathcal{B}}\left(P_{V, \beta}^{\text {odd }}\right)^{*}$. First, consider the case that $v_{\alpha}>0$ for all $\alpha \in V$. Since $V$ is affinely independent, Lemma 4.13 gives a $\mathbf{p} \in \mathbb{R}_{>0}^{n}$ and a $\tau \in \mathbb{R}_{>0}$ with $v_{\alpha}=\tau \mathbf{p}^{\alpha}$ for all $\alpha \in V$. For $\beta \in(\mathcal{A} \cup \mathcal{B}) \backslash V$ set $\lambda^{(\beta)}:=\lambda(V, \beta)$ and observe that by Lemma 3.6,

$$
\left|v_{\beta}\right| \leq \prod_{\alpha \in V} v_{\alpha}^{\lambda_{\alpha}^{(\beta)}}=\tau \mathbf{p}^{\sum_{\alpha \in V} \alpha \lambda_{\alpha}^{(\beta)}}=\tau \mathbf{p}^{\beta},
$$

respectively $\left|w_{\beta}\right| \leq \tau \mathbf{p}^{\beta}$. Hence,

$$
\begin{aligned}
(v, w)(f) & =\sum_{\alpha \in V} v_{\alpha} c_{\alpha}+\sum_{\alpha \in \mathcal{A} \backslash V} v_{\alpha} c_{\alpha}+\sum_{\beta \in \mathcal{B}} w_{\beta} d_{\beta} \\
& \geq \sum_{\alpha \in V} v_{\alpha} c_{\alpha}-\sum_{\alpha \in \mathcal{A} \backslash V}\left|v_{\alpha} c_{\alpha}\right|-\sum_{\beta \in \mathcal{B}}\left|w_{\beta} d_{\beta}\right| \\
& \geq \sum_{\alpha \in V} \tau \mathbf{p}^{\alpha} c_{\alpha}-\sum_{\alpha \in \mathcal{A} \backslash V}\left|\tau \mathbf{p}^{\alpha} c_{\alpha}\right|-\sum_{\beta \in \mathcal{B}}\left|\tau \mathbf{p}^{\beta} d_{\beta}\right| .
\end{aligned}
$$

Therefore, the hypotheses (2) and (3) imply that

$$
(v, w)(f) \geq \tau f(\mathbf{p}) \geq 0 .
$$

In the case $v_{\alpha}=0$ for some $\alpha \in V$, continuity of the mapping in (6) implies $(v, w)(f) \geq 0$ as well. Altogether, $f \in C^{* *}=C \subseteq C_{\mathcal{S}}(\mathcal{A}, \mathcal{B})$. 
4.5. Approximating non-negative polynomials by SONC polynomials. Unlike the situation with sum of squares polynomials, not every non-negative univariate polynomial is a SONC polynomial. However, in this section we show that non-negative univariate polynomials can at least be approximated by SONC polynomials.

For a univariate polynomial $f=\sum_{i=0}^{d} c_{i} x^{i}$ we set

$$
\hat{f}:=c_{0}-\sum_{i=1}^{d-1}\left|c_{i}\right| x^{i}+c_{d} x^{d} .
$$

This is very similar to the SAGE-representative of a polynomial considered in [21, Section 5].

Theorem 4.14. Let $f=\sum_{i=0}^{d} c_{i} x^{i} \in \mathbb{R}[x]$ be a univariate polynomial of even degree $d$ with positive constant coefficient. Let $x_{0}:=\inf \left\{x \in \mathbb{R}_{+} \mid \hat{f}(x)<0\right\}$.

(1) If $x_{0}=+\infty$ (i.e., if $\hat{f}(x) \geq 0$ for all $x \in \mathbb{R}$ ), then $f$ is a SONC polynomial.

(2) Otherwise, there exists a sequence $\left(p_{N}\right)_{N} \subseteq \mathbb{R}[x]$ of SONC polynomials which converges to $f$ uniformly on every compact subset of the open interval $\left(-x_{0}, x_{0}\right)$.

Note that the $p_{N}$ have the property that $\operatorname{deg} p_{N} \rightarrow \infty$ for $N \rightarrow \infty$. Part (1) is essentially a special case of Proposition 4.12, which itself has been obtained before by de Wolff and Iliman [13, Theorem 5.5], see also [21, Theorem 10]. Hence, only part (2) is new. It can be seen as a univariate SONC analogon of the (even multivariate) approximation result in terms of sum of squares polynomials by Lasserre and Netzer (see [18, 19]), where our result also has a restriction to $\left(-x_{0}, x_{0}\right)$.

Proof. For part (1), note that $\hat{f}$ is non-negative on $\mathbb{R}_{+}$if and only if it is non-negative on $\mathbb{R}$. Moreover, it satisfies the hypothesis of Proposition 4.12, and thus $x_{0}=\infty$ implies that $\hat{f}$ is a SONC polynomial. Moreover, Proposition 4.12 implies that $\hat{f}$ can be written as a sum of circuit polynomials using only the constant term and the highest term as outer exponents. Since a non-negative circuit polynomial with negative inner coefficient remains non-negative under flipping the sign of the inner coefficient, $f$ is a SONC polynomial as well.

It remains to show part (2). As in the proof of Theorem 4.11, we use the shorthand notation $C_{\mathcal{S}}(r)$ for $C_{\mathcal{S}}(\{0,1, \ldots, r\} \cap 2 \mathbb{N},\{0,1, \ldots, r\} \backslash 2 \mathbb{N})$ for $r \in \mathbb{N}$. Since the two parameters of $C_{\mathcal{S}}$ are disjoint sets, we shortly write elements in the dual cone as $v$ rather than $(v, w)$, by slight abuse of notation. For $N>d$ define

$$
p_{N}:= \begin{cases}f+\frac{c^{*}}{x_{0}^{N}} x^{N} & \text { for } N>d \text { even } \\ p_{N-1} & \text { for } N>d \text { odd }\end{cases}
$$

for some constant $c^{*}>0$. It is immediately clear that if $|x|<x_{0}$, then $f(x)-p_{N}(x)=c^{*}\left(x / x_{0}\right)^{N}$ converges to zero for $N \rightarrow \infty$, and we even have uniform convergence on every compact subset of $\left(-x_{0}, x_{0}\right)$. It remains to find a suitable value of $c^{*}$ such that $p_{N}$ is a SONC polynomial.

We claim that $v(f) \geq m\left(v_{0}+x_{0}^{-d} v_{d}\right)$ for every $v \in C_{\mathcal{S}}(d)^{*}$, where $m$ is the minimum of the function $v \mapsto v(f)$ on the set

$$
K:=\left\{\frac{v}{v_{0}+x_{0}^{-d} v_{d}} \mid v \in C_{\mathcal{S}}(d)^{*} \backslash\{0\}\right\}=\left\{v \in C_{\mathcal{S}}(d)^{*} \mid v_{0}+x_{0}^{-d} v_{d}=1\right\} .
$$


To prove the claim, consider a fixed $v \in K$ and observe that $v_{d} \geq 0$ because $d$ is even. The definitions of $K$ and $x_{0}$ imply $v_{0} \leq 1$ and $v_{d} \leq x_{0}^{d}$. For $1 \leq i<d$, Theorem 3.5(c) applied on the circuit with outer exponents $0, d$ and inner exponent $i$ then gives

$$
\left|v_{i}\right| \leq v_{0}^{1-i / d} v_{d}^{i / d} \leq 1 \cdot\left(x_{0}^{d}\right)^{i / d} \leq x_{0}^{i} .
$$

Hence, the non-empty set $K$ is bounded and compact. It follows that the function $v \mapsto v(f)$ attains the minimum value $m$ on $K$. This implies the claim.

Since for $N>d$ every element of $C_{\mathcal{S}}(N)^{*}$ can be truncated to obtain an element of $C_{\mathcal{S}}(d)^{*}$, it follows that the auxiliary claim also holds for every $v \in C_{\mathcal{S}}(N)^{*}$. We set $c^{*}:=2|m|$ and it remains to show that for this value of $c^{*}$ our candidate $p_{N}$ is a SONC polynomial. For this, it is sufficient to show that for $N>d$ we have $v\left(p_{N}\right) \geq 0$ for all $v \in C_{\mathcal{S}}(N)^{*}$, and we may assume that $N$ is even. We distinguish two cases.

Case $v_{d} \leq x_{0}^{d} v_{0}$. The inequalities defining $C_{\mathcal{S}}(N)^{*}$ imply that $\left|v_{i}\right| \leq v_{0}^{1-i / d} v_{d}^{i / d}$ for $0 \leq i \leq d$. Using this, we derive

$$
\begin{aligned}
v\left(p_{N}\right) & =\sum_{i=0}^{d} v_{i} c_{i}+v_{N} \frac{c^{*}}{x_{0}^{N}} \geq v_{0} c_{0}-\sum_{i=1}^{d-1}\left|v_{i} c_{i}\right|+v_{d} c_{d}+v_{N} \frac{c^{*}}{x_{0}^{N}} \\
& \geq v_{0} c_{0}-\sum_{i=1}^{d-1} v_{0}^{1-i / d} v_{d}^{i / d}\left|c_{i}\right|+v_{d} c_{d}+v_{N} \frac{c^{*}}{x_{0}^{N}}
\end{aligned}
$$

If $v_{0}=0$, then this expression is non-negative since both $v_{d}$ and $v_{N}$ are. Otherwise, we continue as follows:

$$
\begin{aligned}
v\left(p_{N}\right) & =v_{0}\left(c_{0}-\sum_{i=1}^{d-1}\left(\frac{v_{d}}{v_{0}}\right)^{i / d}\left|c_{i}\right|+\left(\frac{v_{d}}{v_{0}}\right)^{d / d} c_{d}\right)+v_{N} \frac{c^{*}}{x_{0}^{N}} \\
& =v_{0} \hat{f}\left(\left(\frac{v_{d}}{v_{0}}\right)^{1 / d}\right)+v_{N} \frac{c^{*}}{x_{0}^{N}} \geq 0
\end{aligned}
$$

for any $c^{*} \geq 0$ by the choice of $x_{0}$.

Case $v_{d}>x_{0}^{d} v_{0}$. By Theorem 3.5(c) applied on the circuit with outer exponents $0, N$ and inner exponent $d$, we have $v_{N} \geq v_{0}^{-(N-d) / d} v_{d}^{N / d}$, so that using the hypothesis of the current case twice gives

$$
v_{N}>\left(x_{0}^{-d} v_{d}\right)^{-(N-d) / d} v_{d}^{N / d}=x_{0}^{N-d} v_{d}>\frac{x_{0}^{N}}{2}\left(v_{0}+x_{0}^{-d} v_{d}\right)
$$

Since $v\left(p_{N}\right)=v(f)+v_{N} \frac{c^{*}}{x_{0}^{N}}$, employing the auxiliary claim as well as (20) we can conclude

$$
\begin{aligned}
v\left(p_{N}\right) & \geq\left(v_{0}+x_{0}^{-d} v_{d}\right) m+\frac{x_{0}^{N}}{2}\left(v_{0}+x_{0}^{-d} v_{d}\right) \frac{c^{*}}{x_{0}^{N}} \\
& =\left(v_{0}+x_{0}^{-d} v_{d}\right) \cdot(m+|m|) \geq 0
\end{aligned}
$$

As a corollary of the theorem, we see that we can also approximate in the $(x)$-adic topology: 
Corollary 4.15. Let $f \in \mathbb{R}[x]$ be a univariate polynomial with $f(0)>0$. Then for each $N \geq 0$ there exists a $S O N C$ polynomial $p_{N} \in \mathbb{R}[x]$ such that

$$
f \equiv p_{N} \bmod x^{N} .
$$

Proof. If the degree of $f$ is odd, then we may consider $f$ as a polynomial of higher degree with leading coefficient 0 , which has even degree. The hypothesis $f(0)>0$ implies that the $x_{0}$ of Theorem 4.14 exists and is positive, hence we may consider the sequence $p_{N}$ from that theorem. From its construction in the proof of Theorem 4.14, it is clear that it satisfies our claim.

\section{OUTLOOK AND OPEN PROBLEMS}

We have introduced the $\mathcal{S}$-cone as a unified framework for the classes of SAGE and SONC polynomials, provided characterizations of its dual cone and presented several new and several improved results associated with the dual viewpoint. The $\mathcal{S}$-cone exhibits a prominent computationally tractable class within the class of sparse non-negative polynomials. For further computational aspects building upon the projection-free descriptions of the dual cones from Section 3, we refer to the subsequent work of the second author together with Dressler, Heuer and de Wolff [6].

It remains a future task to further understand the relation of the $\mathcal{S}$-cone and its specializations to the underlying class of all non-negative functions (in some special cases polynomials), both from the primal and the dual point of view. Specifically, the relation of the SONC cone to the cone of sparse non-negative polynomials and the dual SONC cone to sparse moment cones (as studied by Nie [22]) deserve further study. It is an open question whether SONC polynomials are dense inside the non-negative ones.

Moreover, since by the results in Section 4.3, the analogue of Putinar's Positivstellensatz already fails in the univariate case, it also remains a challenge to provide computationally attractive types of Positivstellensätze for the $\mathcal{S}$-cone and its specializations.

Acknowledgment. We thank the anonymous referees for their helpful suggestions.

\section{REFERENCES}

1. A. A. Ahmadi and A. Majumdar, Some applications of polynomial optimization in operations research and real-time decision making, Optimization Letters 10 (2016), no. 4, 709-729.

2. - DSOS and SDSOS optimization: more tractable alternatives to sum of squares and semidefinite optimization, SIAM J. Appl. Algebra Geom. 3 (2019), no. 2, 193-230. MR 3939321

3. G. Averkov, Optimal size of linear matrix inequalities in semidefinite approaches to polynomial optimization, SIAM J. Appl. Algebra and Geometry 3 (2019), no. 1, 128-151.

4. V. Chandrasekaran and P. Shah, Relative entropy relaxations for signomial optimization, SIAM J. Optim. 26 (2016), no. 2, 1147-1173.

5. — Relative entropy optimization and its applications, Math. Program., Ser. A 161 (2017), no. 1-2, $1-32$.

6. M. Dressler, J. Heuer, H. Naumann, and T. de Wolff, Global optimization via the dual SONC cone and linear programming, Proc. 45th International Symposium on Symbolic and Algebraic Computation, 2020, pp. $138-145$.

7. M. Dressler, S. Iliman, and T. de Wolff, An approach to constrained polynomial optimization via nonnegative circuit polynomials and geometric programming, J. Symbolic Comput. 91 (2019), 149-172. 
8. M. Dressler, A. Kurpisz, and T. de Wolff, Optimization over the Boolean hypercube via sums of nonnegative circuit polynomials, 43rd International Symposium on Mathematical Foundations of Computer Science, LIPIcs. Leibniz Int. Proc. Inform., vol. 117, Schloss Dagstuhl, 2018, pp. Art. No. 82, 17. MR 3854037

9. M. Dressler, H. Naumann, and T. Theobald, The dual cone of sums of non-negative circuit polynomials, To appear in Adv. Geom., 2020.

10. G. Hall, Optimization over nonnegative and convex polynomials with and without semidefinite programming, Ph.D. thesis, Dept. of Operations Research and Financial Engineering, Princeton University, 2018.

11. G. H. Hardy, J. E. Littlewood, and G. Pólya, Inequalities, Cambridge, at the University Press, 1952, 2nd ed.

12. D. Henrion and A. Garulli (eds.), Positive polynomials in control, Lecture Notes in Control and Information Sciences, vol. 312, Springer, 2005.

13. S. Iliman and T. de Wolff, Amoebas, nonnegative polynomials and sums of squares supported on circuits, Res. Math. Sci. 3 (2016), Paper no. 9.

14. C. Josz, Application of polynomial optimization to electricity transmission networks, Ph.D. thesis, University Paris VI, 2016.

15. O. Karaca, G. Darivianakis, P. Beuchat, A. Georghiou, and J. Lygeros, The REPOP toolbox: Tackling polynomial optimization using relative entropy relaxations, 20th IFAC World Congress, IFAC PapersOnLine, vol. 50(1), Elsevier, 2017, pp. 11652-11657.

16. M. Kojima, S. Kim, and H. Waki, Sparsity in sums of squares of polynomials, Math. Program. 103 (2005), no. 1 , Ser. A, 45-62.

17. J. B. Lasserre, Moments, positive polynomials and their applications, Imperial College Press, London, 2010.

18. J. B. Lasserre and T. Netzer, SOS approximations of nonnegative polynomials via simple high degree perturbations, Math. Z. 256 (2007), no. 1, 99-112.

19. J.B. Lasserre, A sum of squares approximation of nonnegative polynomials, SIAM Rev. 49 (2007), no. 4, $651-669$.

20. V. Magron, G. Constantinides, and A. Donaldson, Certified roundoff error bounds using semidefinite programming, ACM Trans. Math. Software 43 (2017), no. 4, Art. 34, 31.

21. R. Murray, V. Chandrasekaran, and A. Wierman, Newton polytopes and relative entropy optimization, Preprint, arXiv:1810.01614, 2018.

22. J. Nie, The A-truncated K-moment problem, Found. Comput. Math. 14 (2014), no. 6, 1243-1276.

23. M. Putinar, Positive polynomials on compact semi-algebraic sets, Indiana Univ. Math. J. 42 (1993), no. 3, 969-984.

24. C. Riener, T. Theobald, L. Jansson-Andrén, and J. B. Lasserre, Exploiting symmetries in SDP-relaxations for polynomial optimization, Math. Oper. Res. 38 (2013), no. 1, 122-141.

25. R. Schneider, Convex bodies: the Brunn-Minkowski theory, Cambridge University Press, 2014.

26. J. Wang, Nonnegative polynomials and circuit polynomials, Preprint, arXiv:1804.09455, 2018.

27. J. Wang and V. Magron, A second order cone characterization for sums of nonnegative circuits, Proc. 45th International Symposium on Symbolic and Algebraic Computation, 2020, pp. 450-457.

28. J. Wang, V. Magron, and J. B. Lasserre, TSSOS: a moment-SOS hierarchy that exploits term sparsity, Preprint, arXiv:1912.08899, 2019.

29. — Chordal-TSSOS: a moment-SOS hierarchy that exploits term sparsity with chordal extension, Preprint, arXiv:2003.03210, 2020.

30. T. Weisser, J. B. Lasserre, and K.-C. Toh, Sparse-BSOS: a bounded degree SOS hierarchy for large scale polynomial optimization with sparsity, Math. Program. Comput. 10 (2018), no. 1, 1-32.

31. G. M. Ziegler, Lectures on polytopes, Graduate Texts in Mathematics, vol. 152, Springer-Verlag, New York, 1995. 
Lukas Katthän, Helen Naumann, Thorsten Theobald: Goethe-Universität, FB 12 - Institut für Mathematik, Postfach 1119 32, D-60054 Frankfurt am Main, Germany

E-mail address: \{katthaen, naumann, theobald\}@math.uni-frankfurt.de 